\title{
La inmigración árabe en la región de Antofagasta durante el apogeo del salitre (1880-1930): Distribución espacial y redes sociales*
}

\section{Arab immigration to Antofagasta Region during the nitrate boom (1880-1930): Spatial distribution and social networks}

\author{
José Antonio González Pizarro** \\ Marcelo Lufin Varas*** \\ Claudio Llanos Reyes ${ }^{* * * *}$
}

\section{RESUMEN}

Se estudia la presencia de la inmigración árabe en el desierto de Atacama, abarcando la ciudad de Antofagasta y las oficinas salitreras de su hinterland, entre los años 1880-1930. Para tal finalidad, se analiza comparativamente el arribo de palestinos, sirios y libaneses en el continente americano y en Chile, respecto a los factores de su migración, su inserción en las sociedades nacionales, las manifestaciones de xenofobia que padecieron y su impacto en el desarrollo de la cultura y la sociedad. En cuanto a la llegada de esta migración del Medio Oriente en la región de Antofagasta, se ha examinado dos variables, como son la distribución espacial y la funcionalidad de las redes sociales, en base a los Censos Nacionales del periodo, el Archivo de Extranjería del Registro Civil e Identificaciones y el Archivo Histórico del Ministerio de Relaciones Exteriores.

Palabras Claves: Antofagasta, migración, árabes, salitre, censos.

\section{ABSTRACT}

This paper studies Arab immigration to the Atacama Desert, including Antofagasta city and the hinterland nitrate mines in 1880-1930. For this purpose, the arrival of Palestinians, Syrians, and Lebanese at the American continent and Chile is analyzed in terms of migration factors, their insertion in local societies, xenophobia manifestations they faced, and their impact on cultural and societal development. Concerning the arrival of this migration from the Middle East at Antofagasta Region, two variables are examined, that is, spatial distribution and social network functionality, based on the national censuses

\footnotetext{
* El trabajo forma parte del proyecto Fondecyt N¹180319, año 2020.

${ }^{* *}$ Doctor en Historia, Universidad de Navarra. Profesor Titular. Escuela de Derecho-Antofagasta, Facultad de Ciencias Jurídicas, Universidad Católica del Norte, Chile. ORCID: http://orcid.org/0000-0002-4030-0353. Correo electrónico: jagonzal@ucn.cl.

*** Doctor en Economía, Universidad de Illinois. Profesor Asociado. Departamento de Economía-Antofagasta, Facultad de Ciencias Económicas y Administración, Universidad Católica del Norte, Chile. ORCID: https://orcid.org/0000-0002-6856-6401. Correo electrónico: mlufin@ucn.cl.

${ }^{* * * *}$ Doctor en Historia, Universidad de Barcelona. Profesor Adjunto. Instituto de Historia. Pontificia Universidad Católica de Valparaíso. ORCID: https://orcid.org/0000-0002-7612-5497. Correo electrónico: claudio.llanos@pucv.cl.
} 
of that period, the Archive of Foreigners from the Civil and Identification Registrar, and the Historical Archive from the Ministry of Foreign Affairs.

Key words: Antofagasta, migration, arabs, nitrate, censuses

Recibido: febrero 2021

Aceptado: mayo 2021

\section{Introducción}

Durante el periodo que abarcó los años 1880-1930, Gran Bretaña y Francia consolidaron sus imperios. Fue medio siglo donde se verificaron el liberalismo político y económico, la "Paz Armada" hasta el inicio de la primera conflagración mundial. El Imperio Otomano, aliado de Alemania en la Primera Guerra Mundial (1914-1918), perdió su dominio de la Gran Siria (Siria, Líbano, Israel, Palestina, Jordania y unas partes reducidas de Irak y Turquía). Los territorios otomanos fueron ocupados por Gran Bretaña y Francia, mediante la fórmula del Protectorado.

A partir de 1883, Chile, como resultado de la Guerra del Pacífico, configuró un espacio atractivo para la inmigración en las regiones salitreras (Tarapacá y Antofagasta), bajo la égida de la primera globalización del capitalismo. En esta fase llegaron los capitales europeos, el flujo migratorio del Viejo Mundo y las innovaciones de los medios de transporte naviero y de las comunicaciones.

En este encuadre, se verificó la inmigración árabe, dividida bajo el dominio turco y más tarde del protectorado franco-británico. La inmigración del Medio Oriente se planteó con determinados rasgos similares en América Latina y estos se hicieron presentes en Chile y en el norte salitrero del país.

Nuestra hipótesis de trabajo se centra en las oportunidades que ofreció la provincia de Antofagasta, con amplias conexiones internacionales, una potente industria salitrera y un mercado laboral y comercial, que fueron componentes de incentivo para inmigrantes que arribaron de manera mayoritaria desde sus lugares nativos hacia un espacio desértico que no les fue desconocido de modo absoluto, donde pudieron desplegar estrategias de cohesión social como de legitimidad social por medio de sus actividades mercantiles.

El objetivo principal de nuestro estudio es relacionar los elementos constitutivos de nuestra conjetura y señalar de qué modo se realizó el asentamiento de los inmigrantes árabes en Antofagasta.

En nuestro análisis hemos considerado tres aspectos primordiales para abordar el tema en cuestión. Un primer elemento, es el uso de fuentes documentales diversas, desde las diplomáticas (Archivo Histórico del Ministerio de Relaciones Exteriores de Chile), los censos nacionales (Censos Históricos de Población) hasta el repertorio de los prontuarios de extranjería 
de la provincia de Antofagasta (Archivo Histórico de Prontuarios de Identificación para Extranjeros del Registro Civil y de Identificación), que nos permitieron delinear la política migratoria del Estado chileno, la cuantificación de los inmigrantes entre 1895 y 1930, a nivel país y provincial, y compulsar los antecedentes personales de cada uno/a de los/as inmigrantes establecidos en el espacio regional y en la ciudad de Antofagasta. Un segundo aspecto, dice relación con el método empleado donde, además del método histórico para indagar sobre la migración árabe, hemos puesto la atención en el análisis desglosado de los censos nacionales y la aplicación de distintas técnicas y software para el examen de los prontuarios personales, para formular criterios de masculinidad, etarios, distribución al interior de la provincia, relaciones sociales y familiares de localización, las matrices de conexiones de redes sociales, la distribución espacial en Antofagasta y nexos vecinales, los nodos de red y nacionalidad, por medio de diversas tablas y gráficos. Un tercer aspecto, ha sido la construcción sucinta -por razones de espacio- de un marco teórico que sintetizó los diversos paradigmas explicativos del proceso migratorio y el contexto histórico de los flujos migratorios.

Hemos estimado en nuestro análisis hacer breves comparaciones de los flujos árabes y su problemática en determinados países de América Latina.

\section{El mundo del imperio otomano y las nacionalidades agrupadas en la Gran Siria}

Durante el siglo XIX, el Imperio Otomano comenzó a perder fuerza, por varias razones ${ }^{1}$, lo que fue aprovechado por las potencias occidentales. Francia anexionó Argelia -primer país árabe en ser dominado- $y$, posteriormente, auxilió la crisis financiera otomana en la segunda mitad del siglo XIX, convirtiéndose en una de las principales naciones europeas acreedoras de la deuda turca, a través de la OPDA, Ottoman Public Debt Administration².

Entre 1876 y 1910, el Imperio Otomano debió afrontar distintas convulsiones internas derivadas de la promulgación y derogación de la Constitución de $1876^{3}$, la llegada de los "Jóvenes Turcos", una facción del ejército que impulsó diversas obras, la conectividad de la navegación a vapor, los ferrocarriles y el telégrafo, pero también las diferencias al interior del

\footnotetext{
${ }^{1}$ Goodwin, Jason. 2016. Los señores del Horizonte. Una historia del Imperio otomano, Madrid, Alianza Editorial.

2 Rogan, Eugene. 2018. Los árabes. Del Imperio otomano a la actualidad, Barcelona, Editorial Crítica-Planeta.

${ }^{3}$ Alberto Blest Gana, diplomático chileno acreditado en París, redactó un largo informe sobre esta Constitución haciendo notar las presiones ejercidas por las potencias europeas sobre el Sultanato turco, para lograr aplacar las intenciones bélicas de Rusia sobre Constantinopla. Destaca Blest Gana, la "indivisibilidad del Imperio, el Sultán es el Califa de los musulmanes y soberano de todos los otomanos. El islamismo es la religión del Estado, sin ninguna otra distinción ni carácter teocrático, los privilegios religiosos de las comunidades y el libre ejercicio de todos los cultos quedan garantidos. La Constitución proclama la libertad de imprenta, la libertad de enseñanza... la igualdad ante la ley, la admisión a los cargos públicos sin distinción de casta ni religión, el reparto igual de los impuestos... Se instituyen dos Cámaras: de Diputados y de Senadores. La Constitución establece además que la elección se haga por votación secreta. Una ley especial determinará el modo de elegir (a los diputados). Los senadores son nombrados de vida por el Sultán". Archivo Histórico del Ministerio de Relaciones Exteriores [en adelante AHRE]. Oficio de Alberto Blest Gana al ministro de Relaciones Exteriores, Paris, enero 12 de 1877, vol.57.
} 
imperio otomano entre las distintas nacionalidades que convivían ${ }^{4}$. Las medidas de los "Jóvenes Turcos" también significaron la represión contra las provincias árabes, sea cultural, la imposición de la lengua turca en reemplazo del árabe; o administrativa, un reforzamiento del centralismo burocrático, lo que condujo hacia la década de 1910, refiere Rogan, a tener la "inesperada consecuencia de estimular el crecimiento del naciente movimiento nacionalista" 5 .

Los años de 1911 a 1918, el Imperio Otomano debió hacer frente a varios frentes bélicos: la guerra contra Italia por la posesión de Libia y la guerra de los Balcanes ${ }^{6}$. Las pretensiones rusas en la frontera otomana condujeron al imperio otomano, de la mano de Enver Pachá, a alinearse con Alemania contra Inglaterra, Francia y Rusia, en la Primera Guerra Mundial (1914-1918).

La represión llevada por los turcos en Siria en 1915, el reclutamiento forzoso de miles de jóvenes y la confiscación a los campesinos de sus productos para alimentar al ejército, despertó el sentimiento nacionalista contra la opresión y el subdesarrollo bajo los otomanos. Paralelamente, entre los años 1915-1917, Gran Bretaña con Francia firmaron determinados acuerdos para la partición del imperio otomano, al término de la guerra. El acuerdo de SykesPicot o Acuerdo de Asia Menor (Mapa 1), fue un tratado secreto firmado por el Reino Unido y la República Francesa, el 16 de mayo de 1916. El pacto estableció áreas de influencia entre las dos potencias europeas, corroborado en el Tratado de Versalles, que señaló que los países árabes bajo dominación otomana podían ser independientes bajo, acota el especialista británico libanés Albert Houraini, "prestación de ayuda y consejo de un Estado, que asumía el correspondiente 'mandato'. Bajo los términos de los mandatos, otorgados formalmente por la Liga de las Naciones en 1922, Gran Bretaña sería responsable de Irak y Palestina, y Francia, de Siria y Líbano"7. Posteriormente, por medio del Tratado de Lausana, de 24 de julio de 1923, se asignó las fronteras actuales de Turquía, que enfrentó a los nacionalistas turcos contra los aliados, Gran Bretaña, Italia, Francia, Grecia y Armenia, que significó la instauración de la República de Turquía y el fin del Sultanato.

Si esto constituyó sintéticamente la historia político-diplomática entre fines del siglo XIX y primeros lustros del siglo XX, detrás de la fragmentación del Imperio Otomano se registró una historia de similitudes y diferencias entre los países árabes que compusieron la Gran Siria. Posiblemente, el de mayor proyección fue la Declaración de Balfour, del 2 de noviembre de 1917, que estableció un "hogar nacional para el pueblo judío en Palestina" 8 .

\footnotetext{
${ }^{4}$ Rogan, Eugene. 2018. Los árabes, pp. 164-167. Hourani, Albert. 2008. La historia de los árabes, Editorial Zeta, Barcelona.

${ }^{5}$ Rogan, Eugene. 2018. Los árabes, p. 232.

${ }^{6}$ Goodwin, Jason. 2016. Los señores del Horizonte, pp. 443-444.

${ }^{7}$ Hourani, Albert. 2008. La historia de los árabes, p. 388.

8 Krämer, Gudrun. 2006. Historia de Palestina. Desde la conquista otomana hasta la fundación del estado de Israel, Madrid, Siglo XXI, p. 148.
} 
Mapa 1. Acuerdo Sykes-Picot 1916

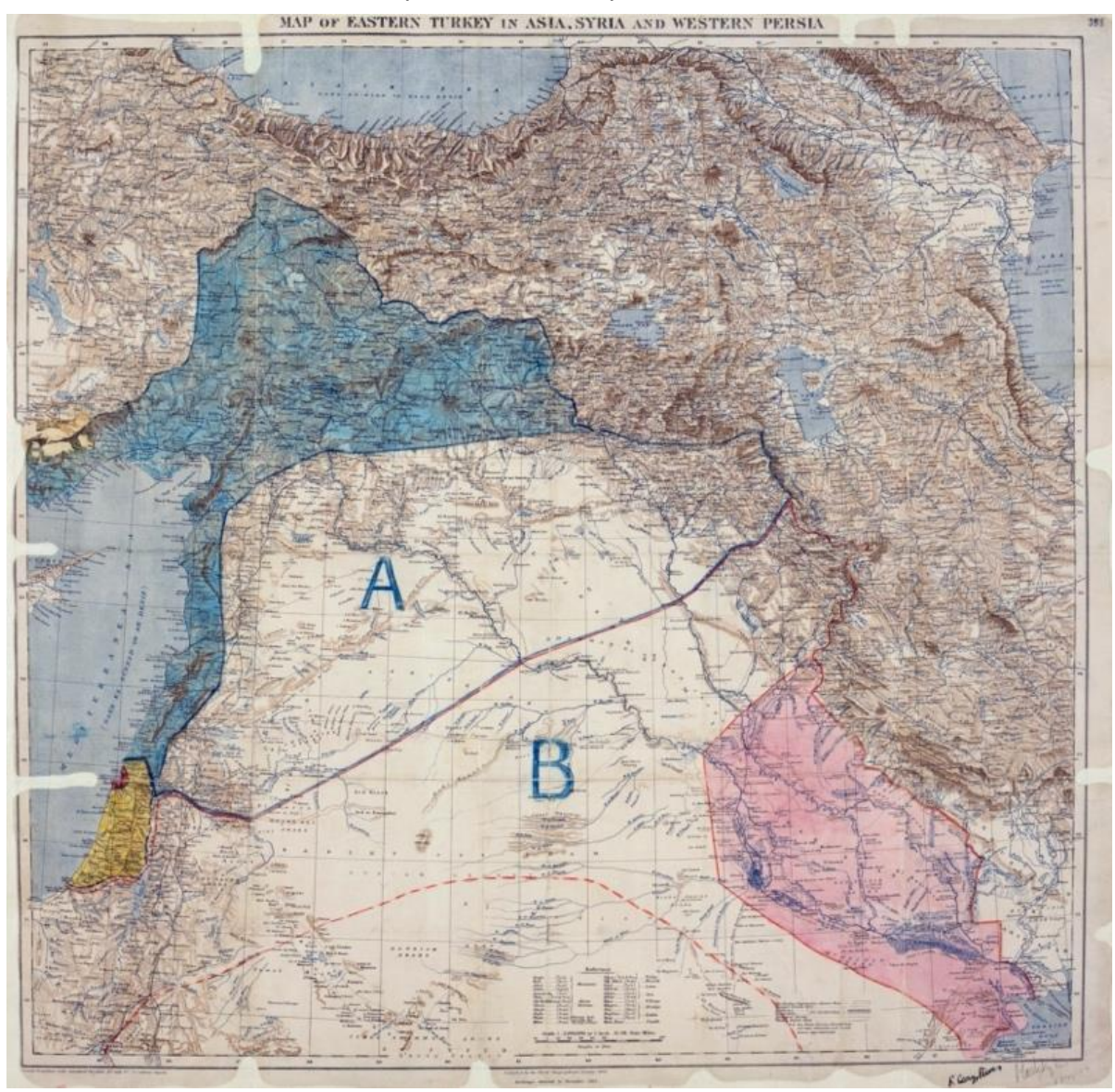

Fuente: Citado de Biblioteca Británica. https://www.bl.uk/britishlibrary/ /media/bl/global/world-warone/collection-item-images/peace-conference-memoranda-respecting-syria-arabia-palestine5.jpg. (A: Francia / B: Británicos) [Consultado el 16 de junio 2021]

Durante la época de la Gran Siria, hacia fines del siglo XIX, el mundo árabe se encontraba regido por el dominio del Sultanato otomano. En los hechos, consigna Hourani, el Sultanato cedió lugar a los señores rurales, cuyo poder radicó en la solidaridad religiosa y tribal, llegando a establecer relaciones directas con los representantes extranjeros. Marruecos estaba bajo dominio franco-español, desde 1860. Egipto era administrado de modo conjunto por británicos y egipcios. Francia dominaba Túnez y Argelia. Italia, desde 1906, Libia. 


\section{La migración árabe hacia el continente americano. Factores y flujos demográficos}

Varios fueron los factores que incidieron en la migración árabe desde la Gran Siria hacia fines del siglo XIX y principios del siglo XX. Los censos otomanos no fueron muy precisos, aunque proclive a catastrar a los hombres jóvenes principalmente con miras a su reclutamiento en el ejército. Se estimó hasta 1931 los censos en base a un "hogar" que se definió como la "familia comensal, incluidas las personas dependientes residentes en un domicilio, como las viudas y los criados, con lo que se evitaba expresamente limitarlos a la familia biológica" ${ }^{9}$. Los pasaportes fueron entregados en función del registro censal -nüfus tezkeresit-, y los individuos asignados a un hogar y éstos a un barrio urbano, una localidad rural y/o una comunidad religiosa. También se debió considerar, en el registro demográfico de la llegada de árabes hacia América, que la mayoría de los "súbditos del imperio no pudieron obtener un pasaporte hasta $1898^{110}$. Los libaneses por ser más independientes pudieron migrar con más facilidad.

El crecimiento demográfico se debió a la eliminación de las epidemias, el hambre y el mejoramiento de los niveles de vida en las ciudades. La mayoría no pudo acceder a la propiedad agrícola. Se calcula que la población de los países árabes hacia 1914 bordeó entre los 35-40 millones de habitantes ${ }^{11}$.

El mundo árabe fue una sociedad dual, escribe Hourani, donde, por un lado, los colonos y ricos comerciantes europeos estaban en la cúspide de la estratificación social, compartiendo los beneficios con los grandes comerciantes e intermediarios colonos de las urbes y, por otro, la mayoría de la población rural estaba alejada de disfrutar las utilidades del febril comercio, al igual que la minoría urbana, excluida de las ganancias de los cambios administrativos y económicos. Hourani apunta al desplazamiento que hubo desde las ciudades mayores hacia los puertos de mar, donde se concentraron los depósitos de mercancías, los bancos y actividades financieras y las reformas urbanísticas y arquitectónicas.

Diversos fueron los factores que motivaron a los árabes de modo indistinto a migrar. No fueron solo las condiciones económicas que posibilitaran abrir camino a un mejor porvenir, huir de los fuertes gravámenes fiscales y del reclutamiento forzoso a los jóvenes. Hubo también disensiones internas por diferencias religiosas y étnicas e incluso por geografías ${ }^{12}$. La migración

\footnotetext{
${ }^{9}$ Krämer, Gudrun. 2006. Historia de Palestina, p. 128.

10 Kukovecz, Gyorgy "The "Turks" of Latin America: remarks of the problems of Syrian- Lebanese emigration", Acta.bibli.u-szeged.hum. Disponible en https://www.semanticscholar.org/paper/The-\%22Turks\%22-of-LatinAmerica-\%3A-remarks-on-the-of-Kukovecz/de4d898e611a4f2b3d33fbd8801fa0d2d87d674c (Consultado el 17 de julio de 2020).

${ }^{11}$ Hourani, Albert. 2008. La historia de los árabes, p. 360.

12 Said, Bahajin. 2008. "El modelo latinoamericano en la integración de los inmigrantes árabes", en Ra Ximhai, Vol. 4, № 3, Ciudad de México, pp. 737-773.
} 
árabe durante el periodo en análisis ha sido abordada a nivel internacional en variados estudios. Fersan clasifica este proceso en varios sub-periodos u olas ${ }^{13}$.

La primera fase fue desde 1880-1914, caracterizada por una fuerte emigración de cristianos maronitas desde Monte Líbano, orientados a Europa, América del Norte, así como Brasil y Argentina. La explicación se debió, según Tarazi-Fawaz ${ }^{14}$, a las regulaciones del Imperio Otomano, el desarrollo de la navegación a vapor y la crisis de la industria de la seda, pues la penetración colonial de occidente en China debilitó la posición de intermediarios de la Gran Siria en este comercio.

La segunda fase fue desde 1915 a 1945, y se produjo bajo el dominio británico y francés en los antiguos territorios de la Siria Otomana. Se dirigió hacia Europa y América del Norte, pero la introducción de cuotas en EE.UU. -para limitar el ingreso de inmigrantes no deseadosprovocó que muchos se desplazaron hacia América del Sur y Australia.

Kemal Karpatha planteado una estadística aproximada para el lapso 1881-1914 de 1,2 millones, donde 600.000 individuos hablantes de árabe provenían desde Siria y del Monte Líbano ${ }^{15}$. A los que se debe agregar un estimado de 100.000 armenios y 250.000 étnicamente mezclados con musulmanes, después de sufrir los armenios masacres continuadas entre 18941896 hasta verificarse el genocidio en el siglo $X X^{16}$.

Stacy Fahrenthold afirmó que, entre "1880 y 1924, una población estimada de medio millón de árabes salió del Imperio Otomano para vivir y trabajar en el continente americano "17.

Se ha indicado que los primeros árabes que emigraron hacia América provinieron desde Marruecos, después de la guerra hispano-marroquí 1859-1860, que tuvo repercusión en América Latina ${ }^{18}$. También del Medio Oriente provinieron judíos y árabes que se establecieron en Brasil, Venezuela ${ }^{19}$. El investigador marroquí Bahajin Said, refiere que los árabes que emigraron hacia América, viajaron con lo mínimo para sustentarse las primeras semanas:

\footnotetext{
13 Fersan, Eliane. 2010. "Syro-Lebanese migration (1880-present): 'push' and "pull' factors", en Working Paper Middle East Institute, Washington DC., USA. Recuperado en https://www.mei.edu/publications/syro-lebanesemigration-1880-present-push-and-pull-factors. (Consultado el 24 de Julio de 2020).

${ }^{14}$ Tarazi-Fawaz, Leila. 1983. "Merchants and Migrants in Nineteenth-Century Beirut", en Harvard Middle Eastern Studies, № 18, Cambridge, pp. 2-3.

15 Karpat, Kemal. 1985. Ottoman population, 1830-1914: demographic and social characteristics, Wisconsin, The University of Wisconsin Press.

${ }^{16}$ Kévorkian, Raymond. 2011. The Armenian genocide. A complete history, London, I. B. Tauris.

17 Fahrenthold, Stacy. 2019. "Arab Labor Migration in the Americas, 1880-1930", American History, in Oxford Research Encyclopedias. Online Publication Date: May 2019.

Recuperado:https://oxfordre.com/americanhistory/view/10.1093/acrefore/9780199329175.001.0001/acrefore9780199329175-e-598 (Consultado 15 de mayo de 2020).

18 González, José. 1989. “España y la guerra con Marruecos, 1859-1860. Política interna, proyección exterior y repercusiones en Iberoamérica”, en Estudios Iberoamericanos, Vol. XV, № 2, Porto Alegre, pp. 369-381.

${ }^{19}$ Said, Bahajin. 2008. "El modelo latinoamericano en la integración", pp.737-773.
} 
"Muchos de ellos despedían a sus familiares con llanto, dejando sus tierras y recuerdos, y viajando a un destino desconocido, soñando con un futuro mejor, sin saber si volverían algún día a encontrarse con ellos o no. No llevaban nada, menos las lágrimas de sus madres, sus brazos para trabajar, un saco de pan y unas pocas monedas que no les servían para nada" ${ }^{20}$.

Sin embargo, la despedida no significó cortar relaciones; por el contrario, hubo remesas de dinero, cartas y motivaciones para que se decidieran otros parientes a migrar.

\section{La migración árabe y su inserción en América y en Chile: de la xenofobia inicial a la legitimidad social mediante el comercio y su contribución cultural}

La llegada de los árabes, independiente de su adscripción religiosa o de su procedencia geográfica específica, fue asumida con recelo en América Latina y en Chile. Hubo una discriminación que se fundó tanto en la ignorancia de lo que significó el Medio Oriente y el Imperio Otomano como un racismo a sus formas de vestir y hablar. Inicialmente a todos/as se les consideró de la religión del islam. El vocablo de "Turcos" fue el adjetivo que aunó a todos los procedentes del Imperio Otomano. Para algunas etnias constituyó un insulto al no ser árabes o musulmanes, como los armenios, o no profesar tal religión, como los cristianos maronitas del Líbano. O constituyó un vejamen confundir como árabes a los sirios con los turcos. La expresión de "Turcos" siguió aplicándose para apuntar a los inmigrantes árabes aun cuando el Imperio Otomano había desaparecido ${ }^{21}$.

Dentro de esta realidad, cabe indicar las reacciones que tuvieron las distintas nacionalidades árabes, frente a la disolución del dominio otomano o estar bajo los protectorados inglés o francés. Algunos se identificaron con cierta plenitud con el Imperio Otomano; otros en las diferencias entre palestinos y libaneses, no faltando las disensiones al interior de una misma etnia. En el caso del Líbano, los conflictos entre drusos y maronitas, pudieron detenerse durante el Imperio Otomano, entre 1860 hasta $1922^{22}$

Las dificultades en el Líbano entre los drusos y los cristianos e incluso la postura de un sector de libaneses de considerarse más occidentales que árabes, gravitaron en decisiones de migrar, aun cuando las condiciones económicas no eran tan desfavorables, como ha estudiado Akran Fouad Khater ${ }^{23}$. En tal sentido, Steven Hyland Jr., ha reparado en las dificultades de englobar a

\footnotetext{
20 lbíd., p. 744.

${ }^{21}$ Klich, Ignacio y Lesser, Jeffrey. 1996. "Introduction 'Turco' immigrants in Latin America”, en The Americas, Vol.53, $\mathrm{N}^{\circ} 1$, jul, Cambridge, pp. 1-14.

22 Akarlı, Engin. 1993. The long peace: Ottoman Lebanon, 1861-1920, Berkeley, University of California Press.

${ }^{23}$ Khater, Akram Fouad. 2001. Inventing home: emigration, gender, and the middle class in Lebanon, $1870-1920$. Oakland, University of California Press. Examina varias perspectivas del conflicto, llamando la atención como determinados historiadores han planteado que los "libaneses originales" eran los marada, o bien eran los maronitas, que se aliaron con la marada en su oposición contra los "árabes". Un autor, Paul Njeim, bajo el seudónimo de Paul Jouplain, sostuvo en 1908, que el Líbano era espiritual e intelectualmente vinculado con el "Oeste", es decir, con
} 
todo el mundo árabe, durante el proceso migratorio, acogiendo las observaciones de Jeffrey Lesse y Raanan Rein, en orden a que "el análisis de los grupos étnicos en general ignora las divisiones intraétnicas que son frecuentemente replicadas en el transcurso de muchas generaciones" ${ }^{24}$. En esta perspectiva, se ha destacado la situación especial de los libaneses y el rol que jugó en su sociedad las misiones cristianas. Todo ello, condujo, por ejemplo, a que Hyland Jr, pueda resumir lo variopinto de la inmigración desde la Gran Siria en Argentina, donde los credos religiosos están subdivididos en su interior:

"La emigración hacia Argentina reflejó la diversidad confesional y regional de la Gran Siria; los cristianos hablantes de árabe, judíos y musulmanes habían salido todos de Damasco, Beirut, Homs, Hama y Alepo y sus lugares del interior, y se establecieron en Buenos Aires, Tucumán y otras provincias y territorios por el año 1914. Los cristianos eran principalmente católicos maronitas, cristianos ortodoxos y católicos armenios. Los judíos llegaron principalmente de Damasco, Alepo e incluso Jerusalén. Aunque los sunitas y los alauitas eran más importantes en Tucumán, la comunidad musulmana diversa en Argentina también consistía en chiitas, drusos e ismaelitas" 25 .

En Argentina, hubo amplios sectores de sirios que se identificaron con el Imperio Otomano y procuraron que las relaciones entre el gobierno de Buenos Aires y la embajada turca en Argentina fueran cordiales. La población árabe musulmana en Argentina en las primeras décadas del siglo XX quedó invisibilizada, sea por asimilarse a los cristianos libaneses o por no verse hostilizados por ser árabes. Se considera que hacia 1907, en Buenos Aires, había 15.000 musulmanes sirio libaneses frente a los 30.000 cristianos $^{26}$. La Primera Guerra Mundial creó tensiones internas en la colonia árabe identificada con el Imperio Otomano, a medida que las

Occidente en vez del "Este". Khater acota, "este mito se ha transformado en una parte elemental de la actual identidad maronita y continúa saliendo a la superficie". El libro de Khater, Inventing Home: Emigration, Gender, and the Middle Class in Lebanon, 1870-1920, se encuentra disponible-recuperado http://ark.cdlib.org/ark:/13030/ft9d5nb66k/ (Consultado el 14 de junio de 2020).

${ }^{24}$ Hyland Jr, Steven.2011. "'Arisen from deep slumber': transnational politics and competing nationalisms among syrian immigrants in Argentina, 1900-1922", en Journal of Latin American Studies, N 43, Cambridge, pp. 547-574. Cita en p.551.

25 Ibíd., p. 553. Una visión general de los inmigrantes árabes en el norte de Argentina, lo brinda Hyland en su tesis doctoral, Hyland Jr, Steven L.2011a. Margins of the Mahjar: arabic-speaking immigrants in Argentina, 1880-1946, Ohio, The Ohio State University. Recuperado en https://etd.ohiolink.edu/!etd.send_file?accession=osu1306510917\&disposition=inline (Consultado el 18 de julio de 2020).

${ }^{26}$ Klich, Ignacio. 1993. "Argentina-Ottoman. relations and their Impact in Immigrants from the Middle East: a history unfulfilled expectations, 1910-1915”, en The Americas, Vol. 50, № 2, Cambridge, pp. 177-205. 
ideas nacionalistas fueron emergiendo en el área del conflicto y se conocieron en las principales ciudades argentinas ${ }^{27}$.

En el caso de México, la llegada de los árabes se dio principalmente durante el Porfiriato, donde no hubo discriminación o acciones racistas oficiales, como aconteció con los chinos. Se ha subrayado que favoreció a la inmigración árabe, el hecho que la mayoría se haya identificado como libaneses y por ende como católicos a secas, en un país donde la veneración por la Virgen de Guadalupe, es transversal a todos los sectores. Tanto los libaneses como los sirios, refiere Theresa Alfaro-Velcamp, al declararse como católicos -fuese cierto o no-pudieron superar una valla para la asimilación a la sociedad mexicana, aunque el periodo post-revolucionario fue complejo para esta inmigración, que gracias a sus negocios y sus enlaces matrimoniales pudieron granjearse un lugar en la sociedad mexicana ${ }^{28}$. Ha sido Hyland el que ha analizado las dificultades interpersonales en la comunidad árabe, exhibida en los tribunales de justicia, donde los años de lejanía entre miembros de una familia, principalmente entre marido y mujer, al reencuentro en América, suscitó quiebres dolorosos, vinculados no solo con violencia intrafamiliar, divorcio y demanda de partición de los bienes conyugales, sino en la trama judicial, donde los documentos traducidos al francés y al español, ocasionaron sospechas. Los más pudientes pudieron plantear sus casos ante la justicia, mientras la mayoría con recursos menguados no pudo exponer sus pleitos en una sociedad distinta a la originaria ${ }^{29}$. El análisis de las sentencias judiciales permitió comprender una serie de acciones delictivas, desde la violación sexual, homicidio, hasta el divorcio. El factor del género gravitó en las desigualdades reservadas en los códigos legales en una situación de una minoría étnica, concluyó Hyland, al estudiar tales circunstancias en el norte de Argentina.

Para la situación de Ecuador, se ha destacado cierta mancomunidad en Palestina entre árabes o palestinos y judíos, por lo que hubo una apertura entre ambas etnias en aquel país, hasta el desencuentro en 1948 con la creación del Estado de Israel, de acuerdo con Solórzano Morán.

Uno de los aspectos más relevantes de la inmigración árabe fue su contribución a la literatura en América Latina. El significativo número de árabes y sus descendientes que redactaron sus testimonios de este trasplante continental ayudó a la comprensión de su migración en cada país ${ }^{30}$. De igual modo, además de su contribución al progreso de actividades

\footnotetext{
${ }^{27}$ Hyland Jr, Steven. 2018. "The Syrian-Ottoman home front in Buenos Aires and Rosario during the First World War", en Journal of Migration History, Vol. 4, № 1, Leiden, pp. 211-235.

${ }^{28}$ Alfaro-Velcamp, Theresa. 2007. So Far from Allah, So Close to Mexico: Middle Eastern Immigrants in Modern Mexico, Austin, University of Texas Press.

${ }^{29}$ Hyland, Steven. 2016. "Arabic-speaking Immigrants before the Courts in Tucumán, Argentina, 1910-1940", en Journal of Women's History, Vol. 28, № 4, Baltimore, pp.41-64.

30 Un panorama general de la contribución a la literatura en América Latina, lo ofrece Saim Houari, M. 2016-2017. Lo árabe en la literatura hispanoamericana: Sergio Macías, Benedicto Chuaqui y Jorge García Usta. These Pour
} 
productivas, la colonia árabe participó -y participa- de las actividades políticas en Argentina, México, Ecuador, Chile, Colombia, por citar algunas naciones ${ }^{31}$.

La resistencia a la llegada de los "Turcos" obedeció, a veces, a la capacidad de su actividad mercantil, una vez superado el recelo sobre su credo religioso, como aconteció en México postrevolucionario. Pero, los inmigrantes no pudieron escapar al ambiente creado por las ideas del darwinismo social, propaladas por Herbert Spencer, y las racistas de Arthur Gobineau y Houston Chamberlain, que consideraron razas inferiores o degeneradas no solo a las africanas, asiáticas $^{32}$ sino también a las del Medio Oriente, especialmente a la árabe.

Tales ideas discurrieron en Chile a través del pensamiento de escritores nacionalistas como Nicolás Palacios, Tancredo Pinochet e incluso del escritor Joaquín Edwards Bello, contrarios a la llegada de extranjeros y, especialmente, los "Turcos" 33 .

El delineamiento de la política migratoria chilena desde el siglo XIX fue incentivar flujos demográficos germánicos o de los países del norte de Europa y, en subsidio, de las naciones meridionales del Viejo Continente. Frente a la inmigración asiática, mantuvo, al igual que el resto de todo el continente americano, una postura de rechazo, imbuida de las ideas racistas en boga. Aquello alcanzó a la población del Medio Oriente, cuando derrotado el Imperio Otomano, se verificó una diáspora árabe hacia América Latina. La comunicación del ministro de Relaciones Exteriores chileno, Conrado Ríos Gallardo, del 16 de diciembre de 1927, fue inequívoca en tal sentido, asumiendo esta visión racista y xenófoba, para obstaculizar la "visación de pasaportes a indeseables". Ríos Gallardo afirmó:

"Este Gobierno, como se habrá U.S. enterado por numerosas declaraciones y publicaciones de prensa, tiene el propósito decidido de conservar, mejorándola en lo posible, la composición de nuestra raza que dio pruebas de vigor y de pujanza.

Una dolorosa experiencia nos ha convencido del error que cometíamos al abrir las puertas de Chile a todas las razas, llegándolo a convertir, no precisamente en asilo contra la

I'obtention du diplôme de Doctorat en Sciences En Langue Espagnole, Orán, Université d'Oran 2. En el caso chileno, Benedicto Chuaqui, un sirio de Homs, abrió la senda de los relatos testimoniales del inmigrante árabe, con su Memoria de un inmigrante, de 1942. Rafide, Matías. 1989. Escritores chilenos de origen árabe, Santiago Instituto Chileno-Árabe de Cultura, pp. 87-110; Samamé, María Olga. 2003. "Transculturación, identidad y alteridad en novelas de la inmigración árabe hacia Chile”, en Signos, Vol. 36, № 53, Valparaíso, pp. 51-73; Cánovas, Rodrigo. 2011. "Letras judías y árabes en Chile: Otros cobijos", en América sin nombre, № 16, Alicante, pp. 131-140.

${ }^{31}$ Solórzano Morán, Claudia. 2015. La migración palestina hacia el Ecuador y su influencia en la sociedad y política exterior ecuatoriana. Trabajo de Investigación para la obtención del título de Internacionalista, Quito, Universidad Internacional del Ecuador; Sanfuentes, Andrés. 1964. La influencia de los árabes en el desarrollo económico de Chile, Memoria de Título, Santiago, Universidad de Chile.

32 González, José. 2020 "Desde la influencia del darwinismo social hasta el imperio de los derechos humanos. Inmigración en Chile entre 1907 y 2018”, en Estudios de Derecho, №169, Antioquía, pp. 325-348.

33 Olguín Tenorio, Myriam y Peña González, Patricia. 1990. La inmigración árabe en Chile, Santiago, Ediciones Instituto Chileno Árabe de Cultura, pp. 104-108. 
opresión, sino en refugio de los perseguidos por la justicia criminal y por la policía sanitaria más celosos de su tranquilidad y progreso. Un somero estudio del cruzamiento de diversas razas nos ha probado que los productos asiáticos y africanos son a menudo inferiores bajo nuestro clima a cada uno de los componentes individualmente considerados. Además, hay nacionalidades que, aparte de sus defectos como troncos de familia, los tiene como simples habitantes del país"34.

En su enumeración surgían los "eslavos que llegan a Chile, judíos en su mayor parte, y de escasa cultura, son revoltosos y sembradores de doctrinas malsanas que han sido causa de desórdenes universitarios y de no pocos disturbios populares...Nada diré a U.S. acerca de las nacionalidades chinas, indostánicas, africanas y otras, ya que el sentimiento público de los países medianamente avanzados las rechaza con elocuente unanimidad".

Y en cuanto a los árabes, el párrafo destinado fue elocuente, de esta mezcla entre racismo y xenofobia difundida por la literatura indicada y en la prensa:

"Los sirios y palestinos, además de ser inadaptables a nuestras costumbres, e inadecuados para formar familia entre nosotros, pertenecen a pueblos del más bajo standard de vida, que les permite competir en forma desleal con las razas superiores. Su comercio preferido es el de ventas de telas y de bisutería ordinarias, ya sea como buhoneros o en tiendas estables. Su falta de honradez en los negocios se ha hecho proverbial y su sistema de liquidarlos por medio del fuego ha llegado a extremos que puede decirse que cerca del $70 \%$ de los incendios que ocurren en el país son provocados intencionalmente por sirios y palestinos" ${ }^{\prime 35}$.

Los oficios que contuvieron noticias de los países árabes estuvieron focalizados principalmente en Egipto, que gozó de mayor autonomía entre las naciones árabes, donde se gestionó la instalación del Consulado General de Chile en El Cairo en 1920. Iniciativa que contó con la oposición del gobierno de Londres, bajo el pretexto de "no otorgar privilegios a los súbditos locales que en virtud de las Capitulaciones todavía en vigor, gozan todos aquellos que ostentan una representación Consular Extranjera" ${ }^{36}$. Chile y Turquía firmaron un Acuerdo de

\footnotetext{
${ }^{34}$ Archivo Histórico del Ministerio de Relaciones Exteriores de Chile (en adelante AHMRE). Conrado Ríos Gallardo, "Sobre visación de pasaportes a indeseables", al Sr. ministro de Chile en Francia. Santiago, 16 de diciem bre de 1927. Confidencial $\mathrm{N}^{\circ} 12$, Vol. 110b.

${ }^{35}$ AHMRE. Conrado Ríos Gallardo, "Sobre visación de pasaportes a indeseables", al Sr. ministro de Chile en Francia. Santiago, 16 de diciembre de 1927, Confidencial N 12, Vol. 110b.

${ }^{36}$ AHMRE. Comunicación del Cónsul General de Chile en Egipto al Ministro de Relaciones Exteriores, El Cairo, 8 de marzo de 1920, Vol. 841-A. Del contenido de la misiva se desprende que el Cónsul de Chile en Egipto era de esa nacionalidad. En diciembre de ese año, le fue reconocida su nobleza. Escribe: "Aprovecho este motive para notificarle que por razón de los vínculos de linaje y raza de nuestra familia con antiguos títulos de nobleza de la Nación Árabe, S.M. El Rey de Hegjaz (sic) se ha dignado rehabilitar estos títulos, confiriendo a mi padre, a mis hermanos y al que subscribe el título de Príncipe para nos y nuestros descendientes. Además S.A. El Sultán de Egipto ha tenido a bien
} 
Amistad el 30 de enero de 1926, convirtiéndose en el primer país latinoamericano en reconocer a la República de Turquía ${ }^{37}$. Hacia fines de 1930, se abrió el primer contacto diplomático entre Chile y Egipto, al firmarse el Tratado de Comercio Chileno-Egipcio, el 5 de marzo de ese año ${ }^{38}$.

Cabe indicar que, disuelto el Imperio Otomano, las nacionalidades definitivas de los árabes inmigrantes hacia el país quedaron a criterio de los protectorados francés y británico. Se podía optar: "Específicamente, en el caso del mandato francés en al-Sham: si se prefería ser sirio o libanés, en el caso del mandato británico, las opciones serían declararse jordano o palestino. Egipto fue un caso excepcional al ser independiente del Imperio otomano desde principios del siglo XIX"39.

La llegada de esta inmigración a Chile estuvo rodeada de un ambiente no propicio a su presencia. La reacción de xenofobia que hemos indicado también fue muy explícita en Chile y ha sido ampliamente estudiada. Las conclusiones han dado cuenta del difícil proceso de integración, la hostilidad y las formas de discriminación que sufrieron, desde los pioneros estudios de Chahuán ${ }^{40}$ y Rebolledo-Hernández ${ }^{41}$. Investigaciones que han proseguido, aunque han tendido a concentrarse en la zona central del país, destacando entre otras, las realizadas por Álvarez-Apse ${ }^{42}$, Cano y Soffia ${ }^{43}$ y Pacheco ${ }^{44}$.

nombrarme Comendador de la Orden del Nilo, orden de las distinguidas de este país. Me permito detallar las condecoraciones del infrascrito como sigue: Comendador del Santo Sepulcro, Comendador de Isabel La Católica; Comendador / Medalla de Oro / de la Cruz Roja Española; Comendador de la Orden del Nilo, Caballero del León y El Sol de Persia; Gran Cruz del Mérito de España". AHRE. Oficio del Cónsul General de Chile en Egipto, al Ministro de Relaciones Exteriores, El Cairo, 9 de diciembre de 1920, Vol. 841-A.

Este mismo Cónsul redactó un detallado informe sobre el Protectorado inglés en Egipto y el particular estatuto del país. AHRE. Oficio del Cónsul General de Chile en Egipto, al Ministro de Relaciones Exteriores, El Cairo, 15 de junio de 1921, vol.893-A. En oficio de 29 de mayo de 1922, el Cónsul informa que Inglaterra ha suprimido el Protectorado y ha reconocido la independencia política de Egipto, instaurándose la monarquía. AHRE, vol. 921-A. Desafortunadamente, la firma del Cónsul es ilegible por lo que desconocemos su identidad.

37 Ministerio de Relaciones Exteriores de Chile. Embajada de Chile en Turquía. Disponible en https://chile.gob.cl/turquia/relacion-bilateral/relaciones-bilaterales.

38 Viera Núñez, Fabiola. 2014. Relaciones diplomáticas de Chile hacia países árabes: casos de Siria y Egipto (20052011). Tesis para optar al Grado de Magister en Estudios Internacionales, Santiago, Universidad de Chile.

${ }^{39}$ Araneda Tapia, Jorge. 2015. "Las ilusiones y los padecimientos de la emigración, el caso de los inmigrantes árabes musulmanes levantinos a Chile 1930-1950”, en Tabula Rasa, № 22, Bogotá, pp. 125-146. Cita en p. 132.

${ }^{40}$ Chahuán, Eugenio. 1983. "Presencia árabe en Chile", en Revista Chilena de Humanidades, № 4, Santiago, pp. 3345.

${ }^{41}$ Rebolledo Hernández, Antonia. 1994. "La "turcofobia". Discriminación antiárabe en Chile. 1900-1950", en Historia, № 28, Santiago, pp. 249-272.

42 Álvarez- Apse Valentina Mar. 2017. La comunidad libanesa en Chile. Un estudio sobre la migración, cultura e identidad de los descendientes libaneses. Tesis de Maestría, Bergen, Universidad de Bergen.

${ }^{43}$ Cano, Verónica y Soffia, Magdalena. 2009. "Los estudios sobre migración internacional en Chile: apuntes y comentarios para una agenda de investigación actualizada", en Papeles de Migración, № 61, Ciudad de México, pp. 129-167.

${ }^{44}$ Pacheco, Juan. 2006. "La prensa árabe en Chile: sueños y realidades árabes en un mundo nuevo". En MEAH, Sección Árabe-Islam, № 55, Granada, pp. 277-322. 
También los trabajos de Agar y Saffie ${ }^{45}$, y Samamé ${ }^{46}$. Otros estudios, como la obra de Mattar ${ }^{47}$ o de Agar ${ }^{48}$, han abarcado análisis de diferentes localidades de Chile. En el caso de Talcahuano, Andrade realizó una indagación de las migraciones árabes durante la primera mitad del siglo $X X^{49}$, y Estrada investigó la situación de la migración árabe de las comunas de Quillota y de Valparaíso ${ }^{50}$.

En el Norte Grande, los árabes han sido estudiados en la región de Tarapacá, con su presencia en Arica ${ }^{51}$ y los nexos con la fe musulmana en lquique ${ }^{52}$. Este sería el primer trabajo en la región de Antofagasta y se diferencia de los anteriores por el uso de las fuentes y la aplicación de nodos de redes familiares y sociales.

Desde una perspectiva teórica más general, en el caso de nuestra investigación, se encuentran antecedentes compatibles con diferentes paradigmas analíticos asociados al proceso migratorio, como por ejemplo las teorías económicas tradicionales (neoclásico) observarían al fenómeno migratorio como un mecanismo de redistribución de fuerza de trabajo, por lo tanto las diferenciales de salarios de renta potenciales y condiciones de vida deberían ser elementos atractores / expulsores asociados a la migración ${ }^{53}$.

Dado el contexto histórico, el caso se puede aproximar también desde un enfoque de Sistema Mundo, que se ajusta mejor a la realidad estudiada, que organiza los lugares dentro de un esquema de jerarquías, en donde algunos ámbitos son centrales y dominantes y otros son espacios subordinados ${ }^{54}$.

\footnotetext{
45 Agar, Lorenzo y Saffie, Nicole. 2005. "Chilenos de origen árabe: la fuerza de las raíces", en MEAH, Sección ÁrabeIslam, № 54, Granada, pp. 3-27.

46

47 Mattar, Ahmad Hassan. 1941. Guía Social de la Colonia Árabe en Chile (Siria-Palestina-Libanesa), Santiago, Imprenta Ahues Hnos.

${ }^{48}$ Agar, Lorenzo. 1983. "El comportamiento urbano de los migrantes árabes en Chile", en Eure, Vol. IX, № 27, Santiago, pp. 73-84.

${ }^{49}$ Andrade Chandía, Javiera. 2018. Inmigración árabe en la primera mitad del siglo XX. Tesis Licenciatura en Historia, Concepción, Universidad Católica de la Santísima Concepción.

${ }^{50}$ Estrada, Baldomero. 2017. "Integración laboral y social de las colectividades árabes en las ciudades medianas de Chile durante el siglo XX: el caso de Quillota", en Historia 396, № 1, Viña del Mar, pp. 59-87; Estrada, Baldomero. 2014. "Desarrollo empresarial inmigrante. La colectividad árabe en Valparaíso, Chile (1900-1940)", en Interciencia, Vol. 39, № 12, Caracas, pp. 850-856.

${ }^{51}$ Díaz Aguad. Alfonso. 2017. "Inmigración árabe en el área Centro Sur Andina" en Alfonso Díaz, Ser Inmigrante entre el Sama y El Loa. Integración económica y social en un espacio de frontera, Arica, ediciones de la Universidad de Tarapacá, pp.15-26.

${ }^{52}$ Crisóstomo Ayres, Stefano. 2018. Espacios, dinámica e integración: el caso de la comunidad musulmana en lquique (1999-2018), Informe de Seminario para optar al grado de Licenciado en Historia, Santiago, Universidad de Chile.

53 Portes, A. \& J. Böröcz. 1989. "Contemporary immigration: theoretical perspectives on its determinants and modes of incorporation", en International Migration Review, Vol. 23, № 3, Los Angeles (CA), pp. 606-630.

${ }^{54}$ Chase-Dunn, C., \& Hall, T. D. 1994. "The historical evolution of world-systems", en Sociological Inquiry, Vol. 64, № 3, Los Angeles (CA), pp. 257-280.
} 
También ciertos elementos son consistentes con el enfoque de sistema de migraciones, donde los flujos de migrantes son solo un aspecto de relaciones más amplias entre países (históricas, culturales, coloniales y tecnológicas), como se aprecia en Fassmann \& Münz ${ }^{55}$, Ghosh $^{56}$, Fawcett \& Arnold $^{57}$.

El caso chileno del periodo corresponde a un fenómeno de expansión productiva con una clara orientación al mercado internacional, que ofreció oportunidades para que diversos colectivos nacionales se instalarán en la zona, habiendo sido atraídos al continente por la posibilidad de instalarse en Brasil y/o Argentina.

\section{La región de Antofagasta en tiempos de la inmigración árabe}

La región de Antofagasta al momento de la llegada del primer flujo de inmigrantes árabes puede caracterizarse en tres dimensiones. En primer lugar las características físicas y el proceso de conectividad entre la costa y su hinterland; un segundo estadio dice relación con el crecimiento poblacional de la región y en los departamentos y la división político-administrativa y, un último nivel, guarda conexión con las dos fases de la explotación minera, que descontando la de la plata del yacimiento de Caracoles, importante en su incidencia regional entre 1870 y 1885 , encontramos los ciclos del salitre, principalmente Shanks entre 1880-1925 y Guggenheim entre 1925-1930, y del cobre, que inició su actividad en Chuquicamata en 1912.

No debió sorprender a los súbditos del Imperio Otomano - de las nacionalidades palestinas, libaneses y sirios-, el desierto de Atacama, pues el conocimiento del paisaje árido era parte de su vivencia, aunque los valles marcaban la diferencia junto a la actividad agrícola y textil. Aun cuando la sequedad y la profunda aridez que inhibe una flora y fauna visibles les debió haber afectado por la inhospitalidad, no fue tan decisivo como para desertar de este espacio, pues se ubicaron en los puertos y desde estos incursionaron hacia la pampa salitrera. Del total de prontuarios examinados, un $10 \%$ ingresó desde Los Andes, proveniente de Argentina, para instalarse en Antofagasta. En lo que se denomina clima árido subtropical, en la clasificación de Köppen, hallamos diferencias entre el clima desértico con nublados abundantes, producido por la presencia del anticiclón del Pacífico, dominante en la costa y el clima desértico normal, que se encuentra traspasada la cordillera de la costa, por sobre los 1.000 metros, que domina la pampa. Se caracteriza por la baja humedad, fuerte oscilación diaria y la carencia absoluta de

\footnotetext{
55 Fassmann, H. \& Münz, R. 1992. "Patterns and trends of international migration in Western Europe", en Population and Development Review, Vol. 18, № 3, Nueva York, pp. 457-480.

56 Ghosh, B. 1992. "Migration, trade and international economic cooperation. Do the interlinkages work?", en International Migration, Vol. 30, №s 3-4, Geneve, pp. 377-398.

57 Fawcett, J.T., \& F. Arnold. 1987. "19: Explaining Diversity: Asian and Pacific Immigration Systems", en Center for Migration Studies, Vol 5, №3, New York, pp. 453-473.
} 
precipitaciones. Ya sobre los 3.000 metros sobre el nivel del mar, es posible percibir los rasgos del clima desértico marginal de altura ${ }^{58}$.

La conectividad discurrió en relación directa con la expansión de la actividad salitrera -hacia el llamado cantón Central o boliviano, entre el Salar del Carmen y el poblado de Sierra Gordaque, la Compañía de Salitres y Ferrocarriles de Antofagasta llevó a cabo desde la década de 1870. El ferrocarril rápidamente amplió su cobertura regional a internacional, al transformarse en la empresa del Ferrocarril de Antofagasta a Bolivia, posibilitando la fundación de la ciudad de Uyuni, en ese país. El nudo de la oficina Salinas, en pleno yermo, aunó la conectividad de las oficinas salitreras con Antofagasta, en la costa, y con Calama y Bolivia hacia el oriente. Más tarde, el surgimiento de los restantes cantones, el de Taltal, el del Toco y el de Aguas Blancas, facilitó la creación de empresas ferroviarias privadas vinculadas con la explotación del nitrato, a partir de la década de 1890. Esto se reflejó en los cambios en la fisonomía portuaria de Antofagasta, Tocopilla y Taltal, desde modestos muelles a una mayor implementación de mecanización de estos hasta su transformación de nuevos molos en el siglo XX. Con la explotación de Chuquicamata, se comenzó la red caminera de asfalto, exigida por la introducción de los camiones, tractores y palas mecánicas ${ }^{59}$.

En cuanto a las localidades costeras y las oficinas salitreras, el crecimiento que experimentaron fue mucho más notorio después de la Guerra Civil de 1891, cuando la estrategia salitrera de las Combinaciones posibilitó un desarrollo progresivo controlando la producción hasta la primera década de 1910.

El crecimiento poblacional marcó un hito en el censo de 1907 cuando la inmigración extranjera alcanzó un $18 \%$ de la población total regional, coincidiendo con la bonanza salitrera del bienio 1906-1907. Una comparación de estos cambios se tiene (Tabla 1) en base de los censos nacionales desde 1885 a 1930.

Tabla 1. Evolución de la población en la provincia de Antofagasta: 1885-1930.

\begin{tabular}{|c|c|c|c|c|c|c|c|c|}
\hline Provincia & \multicolumn{2}{|c|}{ Censo 1895} & \multicolumn{2}{c|}{ Censo 1907} & \multicolumn{2}{c|}{ Censo 1920 } & \multicolumn{2}{c|}{ Censo 1930} \\
\hline $\begin{array}{c}\text { Total } \\
\text { Población }\end{array}$ & Hombres & Mujeres & Hombres & Mujeres & Hombres & Mujeres & Hombres & Mujeres \\
\cline { 2 - 9 } & 26,039 & 18,046 & 71,846 & 41,477 & 99,491 & 72,839 & 101,41 & 77,353 \\
\hline $\begin{array}{c}\text { Total } \\
\text { Población } \\
\text { Extranjera }\end{array}$ & 4,049 & 2,141 & 13,805 & 3,995 & 10,813 & 4,909 & 8,232 & 3,497 \\
\hline
\end{tabular}

Fuente: Elaboración propia en base de los Censos nacionales de 1895, 1907, 1920 y 1930

\footnotetext{
58 Fuenzalida, Humberto. 1950. "Clima”, en Geografía Económica de Chile, Santiago, Imprenta Universitaria, Tomo I, pp. 188-257.

59 Blakemore, Harold. 1996. Historia del Ferrocarril de Antofagasta a Bolivia. 1888-1988, Santiago, Impresos Universitarios S.A.; Thomson, lan. 2003. La Red Norte: la historia de los ferrocarriles del norte chileno, Santiago, Imprenta Silva; González, José. 2008. "La conquista de una frontera. Mentalidades y tecnologías en las vías de comunicación en el desierto de Atacama”, en Revista de Geografía. Norte Grande, N 40, Santiago, pp. 23-46.
} 
En este contexto, se tuvo la presencia de la emigración árabe, que fue más visible a nivel nacional, a partir de 1907, agrupada bajo la denominación de "Turcos", y desde 1920, distinguiendo los sirios y palestinos, continuando un segmento adscribiéndose a turcos hasta 1930.

Cabe indicar que la división política administrativa se tradujo que de los tres departamentos que componía la provincia de Antofagasta, el de Antofagasta, a través de sus subdelegaciones, englobó a toda la pampa salitrera, incluido el cantón central, el más importante en cuanto al número de oficinas salitreras y población; también el cantón de Aguas Blancas, Calama -y Chuquicamata- y toda la precordillera andina, hasta 1925, cuando se creó el Departamento del Loa; los otros departamentos, Tocopilla y Taltal, incluyeron a sus respectivas comunas y cantones salitreros. La población árabe era inexistente hacia 1895 , pues hubo dos súbditos turcos transeúntes. El Censo de 1907, el más explicativo, nos refiere los siguientes datos (Tabla 2).

Tabla 2. Población y religión de inmigrantes del Imperio Turco en 1907.

\begin{tabular}{|c|c|c|c|c|c|c|c|c|}
\hline \multirow{3}{*}{ Departamento } & \multirow{3}{*}{$\begin{array}{c}\text { Hombres } \\
\text { Poblac. } \\
\text { Urbana }\end{array}$} & \multirow{3}{*}{$\begin{array}{l}\text { Mujeres } \\
\text { Poblac. } \\
\text { Urbana }\end{array}$} & \multirow{3}{*}{$\begin{array}{c}\text { Hombres } \\
\text { Poblac. } \\
\text { Rural }\end{array}$} & \multirow{3}{*}{$\begin{array}{c}\text { Mujeres } \\
\text { Poblac } \\
\text { Rural }\end{array}$} & \multirow{3}{*}{ Total } & \multirow{2}{*}{\multicolumn{2}{|c|}{ Religión Musulmana }} & \multirow{3}{*}{ Total } \\
\hline & & & & & & & & \\
\hline & & & & & & Homb. & Mujer & \\
\hline Antofagasta & 188 & 33 & 26 & - & 247 & 210 & 33 & 243 \\
\hline Tocopilla & 5 & 4 & 1 & - & 10 & 6 & 4 & 10 \\
\hline Taltal & 8 & 4 & 9 & 1 & 22 & 13 & 5 & 18 \\
\hline
\end{tabular}

Fuente: Elaboración propia en base de los datos del Censo de 1907.

Es significativo que la totalidad de la inmigración árabe se reconozca religiosamente como musulmana, cuando sabemos que provinieron palestinos, libaneses y sirios, en su mayoría cristianos. En el Censo Nacional de 1920 se desdibuja tal adscripción al credo del islam.

Se debe puntualizar que, en el contexto de los extranjeros en la provincia de Antofagasta, la totalidad del mundo árabe fue minoritaria, incluso en relación a lo que hemos denominado en otro estudio como colonias minoritarias, en el caso europeo, italianos y españoles, con 968 y 1.425 personas, respectivamente ${ }^{60}$.

Los árabes vivieron el auge de la industria salitrera, desde 1907 hasta que comenzó a declinar en la década de 1920, frente a la competencia del nitrato sintético y los costos de la explotación del modelo Shanks. Las diversas crisis que afectaron a la industria se tradujeron en el cierre gradual de oficinas salitreras y el consiguiente despoblamiento de la pampa. Aun

\footnotetext{
60 González, José; Galeno, Claudio; Lufin, Marcelo. 2018. “La migración de las colonias minoritarias europeas y latinoamericanas en el desierto de Atacama. Españoles, italianos, colombianos y ecuatorianos en Antofagasta, 18801930", en Tapia, Marcela y Liberona, Nanette (Editoras), El afán de cruzar las fronteras. Enfoques transdisciplinarios sobre migraciones y movilidad en Sudamérica y Chile. RIL Editores-Universidad Arturo Prat, pp. 223-257.
} 
cuando en 1926 se inauguró la oficina María Elena, bajo el sistema Guggenheim, no pudo remontar la industria salitrera que se vio enfrentada a la crisis mundial de 1930. Distinta fue la fortuna que le cupo a la industria del cobre -con Chuquicamata-, que reemplazó en su aporte al erario fiscal a la industria del salitre ${ }^{61}$.

La crisis del salitre y la mundial, afectó seriamente a las ciudades cabeceras de departamentos en lo económico y social, lo que derivó en un drástico descenso de la población extranjera.

\section{Los árabes en la región de Antofagasta. Elementos descriptivos demográficos básicos.}

Utilizando información de los censos nacionales se pudo analizar los patrones de localización de la población migrante que nos ocupa. En el gráfico 1, se muestra este colectivo, resumiendo la información de varias provincias en Chile.

Gráfico 1. Total de personas de nacionalidad turca, libanesa, palestina según censos por provincias.

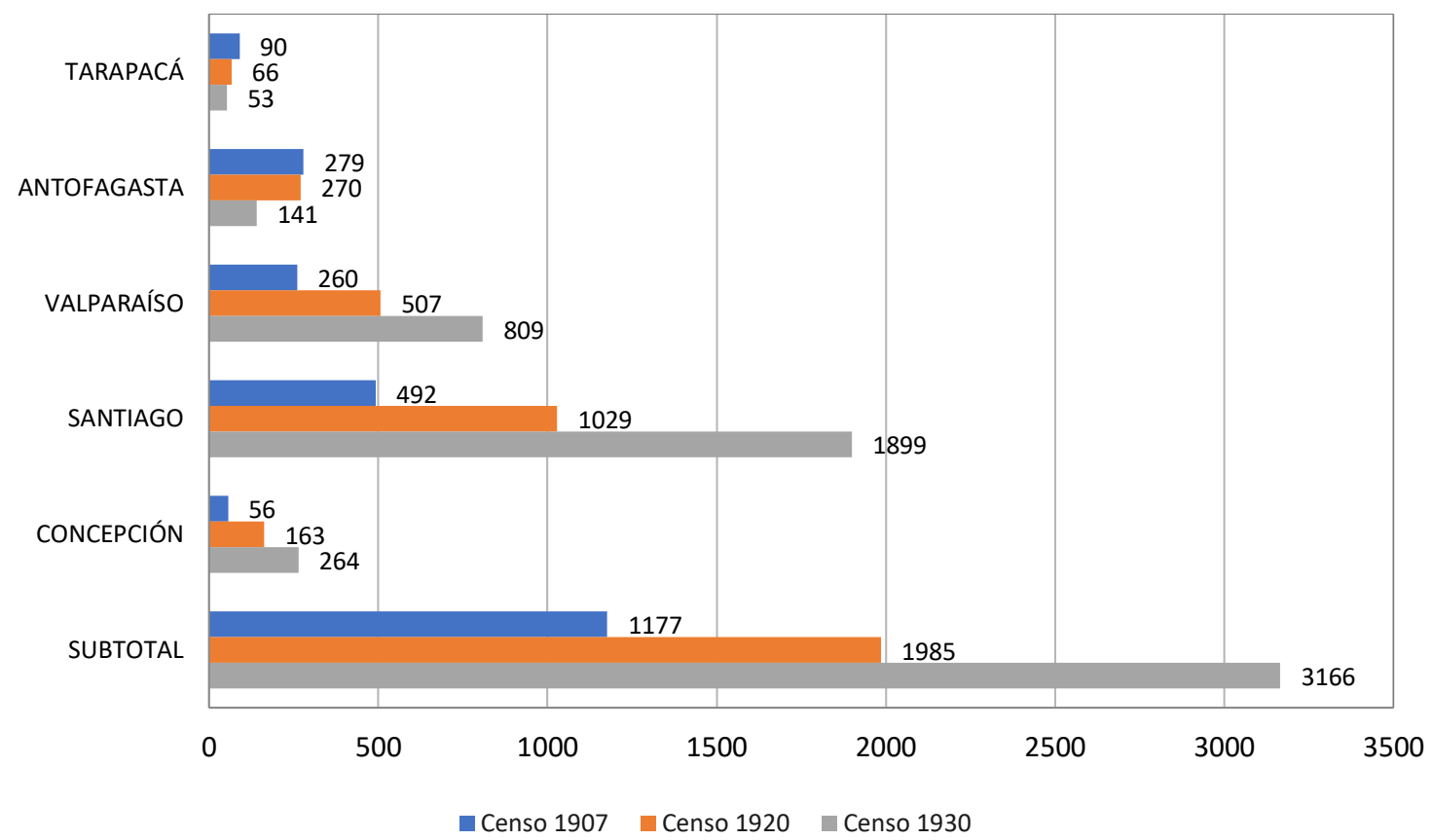

Fuente: Elaboración propia con base a Censos de Población de 1907, 1920 y 1930

La mayor presencia como colectivo se encuentra en las provincias de Santiago, Valparaíso y Concepción. Siendo la Provincia de Antofagasta la que ocupa el 4to lugar en importancia por el

61 González Pizarro, José Antonio. 2016. La épica del salitre en el desierto de Atacama: 1880-1967. Trabajo, tecnologías, vida cotidiana, conflicto y cultura, Saarbrücken, Editorial Académica Española. 
tamaño del colectivo. Los árabes en Antofagasta provinieron directamente desde el Medio Oriente, en base de los datos personales, por lo que podemos conjeturar, en las otras provincias chilenas pudieron ingresar un número significativo desde Argentina, uno de los principales países receptores de esta inmigración en la época de estudio.

Cuando se incorpora la composición por sexos, destaca la alta tasa de masculinización que caracteriza a estos flujos. En la tabla 3 se presentan los índices de masculinidad calculados con base a la información censal histórica.

Tabla 3. Índices de masculinidad (número de hombres / número de mujeres).

\begin{tabular}{|c|c|c|c|}
\hline Provincia & Censo 1907 & Censo 1920 & Censo 1930 \\
\hline Tarapacá & 9,0 & 3,1 & 1,9 \\
\hline Antofagasta & 5,6 & 3,6 & 3,3 \\
\hline Valparaíso & 5,0 & 2,2 & 1,6 \\
\hline Santiago & 2,4 & 1,7 & 1,7 \\
\hline Concepción & 3,0 & 2,0 & 1,4 \\
\hline Subtotal & 3,7 & 2,0 & 1,7 \\
\hline
\end{tabular}

Fuente: Elaboración propia con base a Censos de Población de 1907, 1920 y 1930

El índice de masculinidad refiere una migración donde la mujer no se sumó de modo destacado a este flujo migratorio; situación que fue declinando con el tiempo. Además, la masculinidad fue más alta en las provincias del norte que en la zona centro-sur, idea compatible con el norte como un espacio de frontera, de expansión del límite territorial nacional de Chile. Algunos árabes en Antofagasta, como Sabella o Rumie, desposaron a mujeres chilenas, y su influencia social y económica se evidenció en ser las más significativas joyerías de la región (Hermanos Sabella, Zalaquett), logrando un lugar destacado en la sociedad regional. Andrés Sabella fue el más prominente escritor de ascendencia árabe de la ciudad.

Para entrar en detalle a la situación de este colectivo en la Provincia de Antofagasta, por localidades, la información disponible en los censos nacionales no es suficientemente desagregada, por esta razón se utilizaron los prontuarios del registro civil ${ }^{62}$. El gráfico 2 , contiene la cantidad de casos de interés, según la nacionalidad declarada en el prontuario, encontrados en las diferentes localidades de la provincia.

\footnotetext{
62 Los prontuarios fueron un documento de 8 páginas de antecedentes personales que llevó a cabo la policía con el Servicio del Registro Civil e Identificaciones, donde se recogieron datos como: nacimiento, lugar, padres, nivel de escolaridad, oficio/profesión, nexos familiares en Chile $u$ en otros lugares, relaciones de personas que lo conocen, cómo llegó, cuándo y por donde ingresó, donde vive actualmente, fecha del prontuario, señas físicas, fotografía. Fue resultado del "Convenio celebrado entre las Policías de La Plata i Buenos Aires (Argentina), de Río de Janeiro (Brasil), de Santiago de Chile, de Montevideo (República Oriental del Uruguay), n.o 180, Santiago 18 de enero de 1906" (Anuario, 1909, 239).
} 
Gráfico 2. Personas por nacionalidad y localización en la provincia.

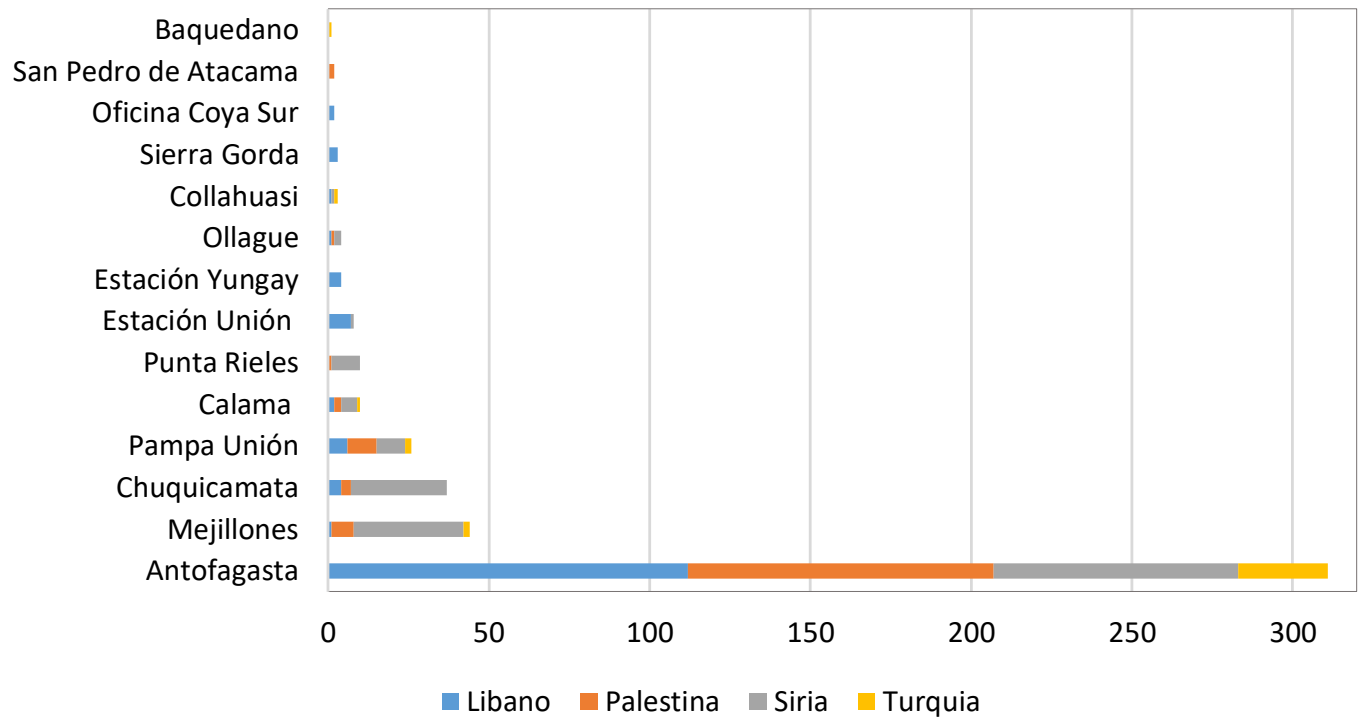

Fuente: Elaboración propia con base a prontuarios del registro Civil. Ingresos declarados al país entre 1880 y 1930

El 61\% de los casos está concentrado en la ciudad de Antofagasta, luego Mejillones (8\%), Chuquicamata (7\%) y Pampa Unión (5\%) aportan un total de $20 \%$ como conjunto. El $34 \%$ de los casos corresponde a sirios, $30 \%$ libaneses y un $28 \%$ de palestinos en el total regional. Las localidades de Antofagasta (43\%), Mejillones (19\%) y Chuquicamata (17\%) concentran la mayor proporción de la población siria en la provincia.

Utilizando los prontuarios también puede evidenciarse la alta masculinización de este colectivo migrante, datos que se representan en el gráfico 3 . En cada grupo nacional la composición está fuertemente masculinizada. La mayor presencia relativa de mujeres de observa en el grupo libanés, que pudo relacionarse con la venida posterior de las mujeres a integrarse al grupo masculino familiar asentado. Las familias sirias y libaneses prosiguieron las costumbres de sus sociedades, donde el carácter patriarcal y endogámico dominó los enlaces u compromisos matrimoniales ${ }^{63}$.

\footnotetext{
63 Nasser, Ghinwa.2015. Inmigración, identidad y estrategias de adaptación a la sociedad receptora. El caso de las mujeres sirias y libanesas en Argentina (primera mitad del siglo XX). Tesis Doctoral. Departamento de Historia de América. Programa de Doctorado en Historia, Literatura y Poder. Procesos interétnicos y culturales en América. Universidad de Sevilla.
} 
Gráfico 3. Índice de masculinidad en base a prontuarios

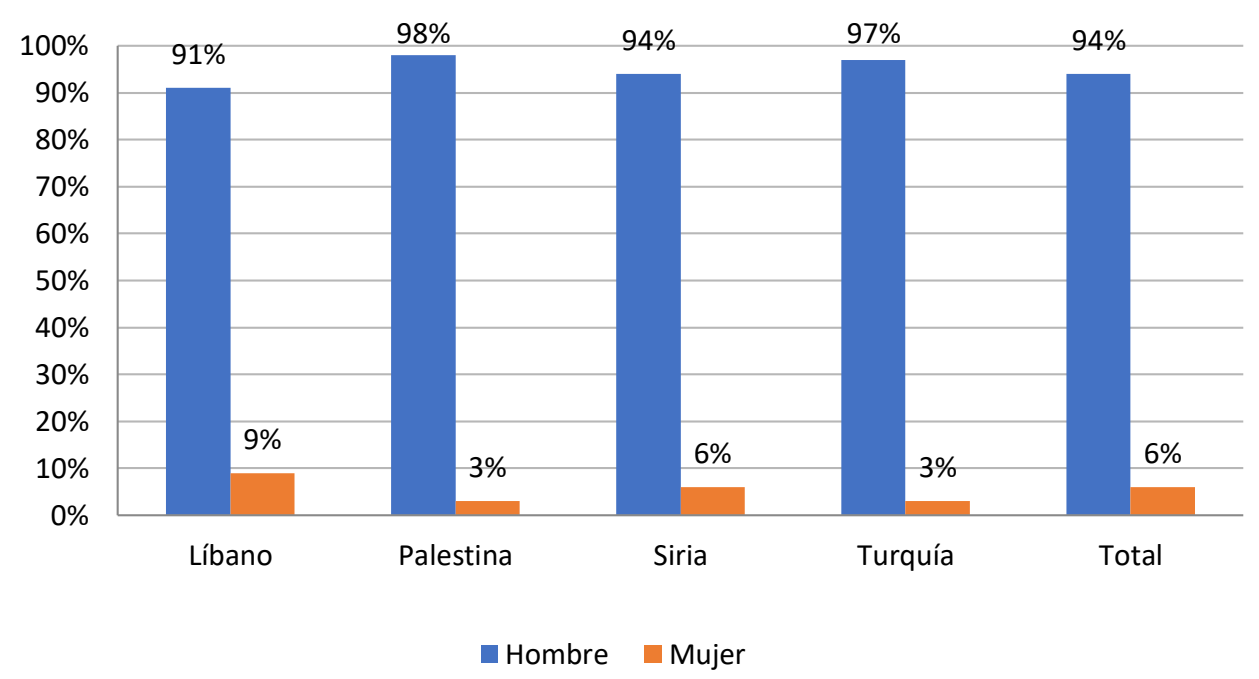

Fuente: Elaboración propia con base a prontuarios del registro Civil. Ingresos declarados al país entre 1880 y 1930

\section{Los árabes en la región de Antofagasta. Centro \& Hinterland: Vinculaciones entre localidades a partir del patrón de conexiones de los residentes}

En los prontuarios las personas declararon su red de conexiones sociales, por medio de sujetos que eran indicados como referencias de antecedentes o conocimiento de ellas, y también por medio de la explicitación de su red familiar de vínculo y la localización de estos individuos en diferentes lugares, incluido el extranjero. Aún cuando el proceso de toma de datos no fue construido desde una perspectiva de red social, la forma de responder de los/as entrevistados se corresponde con un tipo de generación de redes, llamado generador de nombres ${ }^{64}$.

Con base a este principio se construyó entonces una Red de Dos Modos ${ }^{65}$, donde las personas prontuariadas quedan asociadas a un conjunto de lugares, a partir de identificar familiares residiendo en dichas áreas. Así se forma una matriz de $\mathrm{N}$ por $\mathrm{L}$ dimensiones, donde $N$ es el número de prontuarios y $L$ el número de lugares donde residen las personas referidas por cada entrevistado/a. Dado que cada prontuariado está en una localización L, se puede entonces calcular una matriz de un modo LxL dónde la localidades aparecen vinculadas por el

\footnotetext{
${ }^{64}$ Campbell, Karen y Lee, Barret. 1991. "Name generators in surveys of personal networks", en Social Networks, № 13, Laussane, pp. 203-221; Marin, Alexandra y Hampton, Keith, 2007. "Simplifying the personal network name generator", en Field Methods, № 19, Los Angeles (CA), pp. 163-193; Pustejovsky, James y Spillane, James, 2009. "Question-order effects in social network name generators", en Social Networks, № 31, Laussane, pp. 221-229.

65 Borgatti, Stephen, \& Everett, Martin. 1997. "Network analysis of 2-mode data”, en Social Networks, Vol. 19, № 3, Laussane, pp. 243-269.
} 
número de personas que las asocian porque sus residentes señalan a otras personas residiendo en dichos lugares, pero sin recurrir al teorema de la Dualidad de Breiger ${ }^{66}$.

Esta matriz LXL muestra el grado de asociación entre las localidades y puede ser examinada para determinar los grados de centralidad derivados del patrón de conexión de los residentes, lo que posibilita generar una jerarquía de lugares ${ }^{67}$, e incluso formar grupos de localidades basados en sus grados de similitud, mediante, por ejemplo, análisis de conglomerados jerárquicos para identificar comunidades ${ }^{68}$.

Utilizando la matriz de vinculaciones con sus personas relacionadas se obtiene una matriz social entre localizaciones geográficas, que excede el límite de la provincia de Antofagasta, porque las relaciones de las personas se extienden globalmente, de esta manera aparecen las diferentes localidades de la provincia vinculadas, a través de las personas, con diferentes localidades del oriente medio, así como diferentes países.

El gráfico 4, representa el grafo de las localidades vinculadas por medio de las personas, desplegadas en un "layout" construido mediante un algoritmo tipo Kamada-Kawai, esto implica que las localidades más centrales, con mayor conectividad de casos aparecen en la zona central y las comunidades menos mencionadas aparecen en la periferia. En el diagrama el tamaño del nodo refleja la centralidad medida con índice de Eigencetrality.

La forma indica el tipo de localidad: los círculos son localidades en Provincia de Antofagasta, los cuadrados son otras localidades en Chile y los triángulos son localidades en extranjero. El color indica la pertenencia a una comunidad cohesiva, estas se identificaron mediante el algoritmo de Girvan-Newman ${ }^{69}$ y se detectaron solo dos comunidades. El grosor de la línea indica la fuerza de la conexión, número de casos asociados a esta vinculación específica.

En el gráfico 4 se aprecia la importante centralidad de la ciudad de Antofagasta, como centro de una comunidad también integrada por Mejillones, Calama y Chuquicamata y otro centro en Pampa Unión asociado a la segunda comunidad identificada. Repárese la relevancia que

\footnotetext{
66 Breiger, Ronald. 1974. "The Duality of Persons and Groups", en Social Forces, Vol. 53, № 2, Chapel Hill, pp. 181190.

67 Bonacich, Phillip. 2007. "Some unique properties of eigenvector centrality", en Social Networks, Vol. 29, № 4, Laussane, pp. 555-564; Ilyas, M. U., y Radha, H. 2011. "Identifying influential nodes in online social networks using principal component centrality", en 2011 IEEE International Conference on Communications, New York, pp. 1-5.

68 Girvan, Michelle y Newman, Mark. 2002. "Community structure in social and biological networks", en Proceedings of the National Academy of Sciences, Vol. 99, № 12, Washington, pp. 7821-7826; Qiao, Shaojie; Li, Tianrui; Li, Hong; Peng, Jing y Chen, Hongmei. 2012. "A new blockmodeling based hierarchical clustering algorithm for web social networks", en Engineering Applications of Artificial Intelligence, Vol. 25, № 3, Amsterdam, pp. 640-647.

${ }^{69}$ Con relación al método para detectar comunidades, este es un método de aglomeración jerárquico utilizado para detectar comunidades en sistemas complejos. Para esto comienza ordenando los enlaces según su rol en la conectividad estructural de la red (usando un indicador de edge-betweenness) y va secuencialmente eliminándolos, en cada paso se identifican los componentes remanentes en el sistema. Esto genera un dendrograma que se lee desde la red total y sus componentes hacia los nodos individuales completamente separados, pasando por los diferentes subcomponentes identificados en cada etapa.
} 
adquirieron para los árabes aquellas localidades que conjugaron un centro urbano, gran conectividad y posibilidades mercantiles, como fueron Mejillones (desde 1906 base de la mayor Maestranza de ferrocarriles de Chile, perteneciente al del Ferrocarril de Antofagasta a Bolivia, de propiedad inglesa); Antofagasta, por ser puerto principal, asiento de las grandes casas comerciales europeas; Calama por constituir centro de abastecimiento hacia los pueblos precordilleranos y frontera de Bolivia; y Chuquicamata, la floreciente mina de cobre, consolidando su gran campamento/localidad.

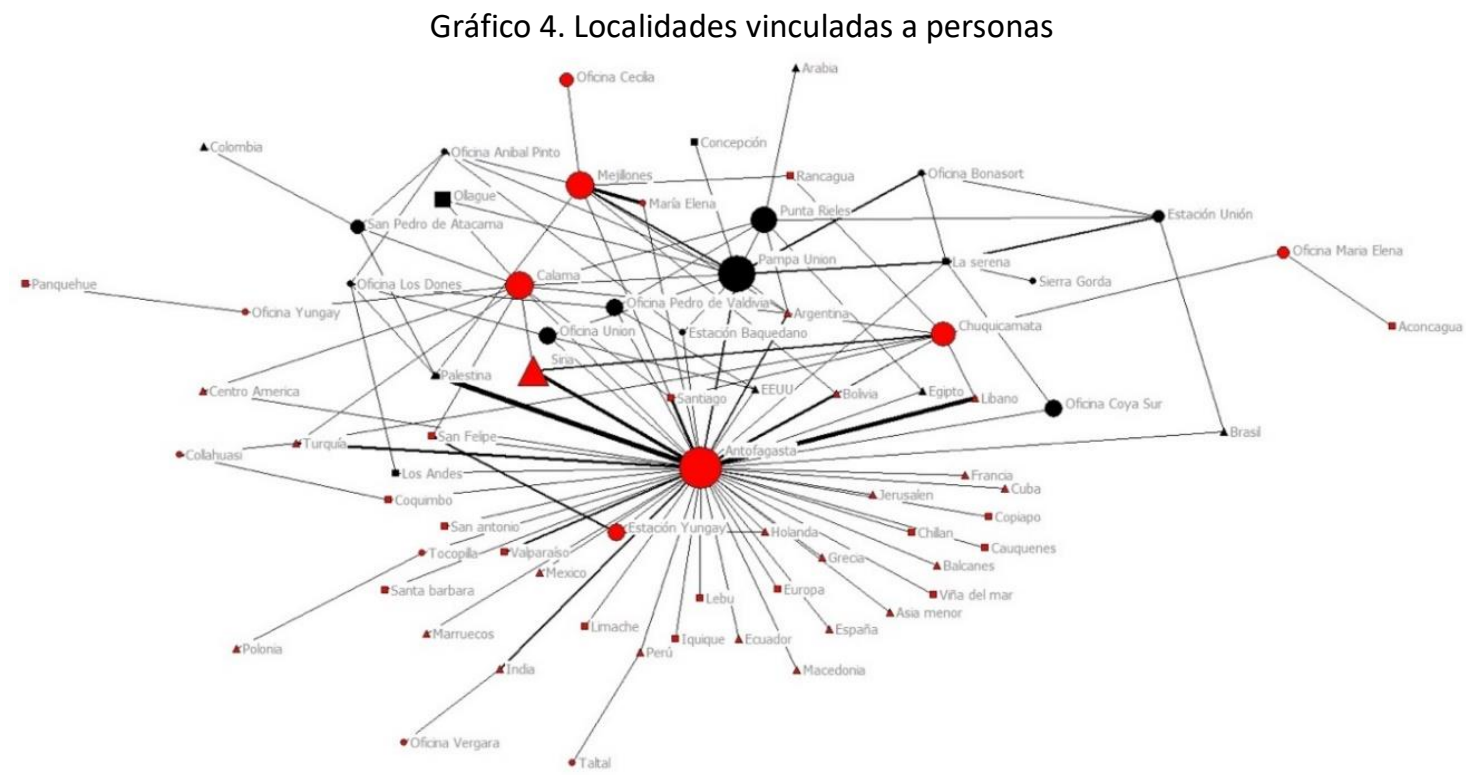

Fuente. Elaboración propia en software Netdraw, a partir de una matriz de localizaciones de los contactos señalados en los prontuarios de los residentes en la Provincia de Antofagasta durante el periodo de estudio.

En el gráfico 5 se ha despejado la información correspondiente a localidades fuera de la Provincia de Antofagasta, para apreciar mejor la configuración de las comunidades, divididos entre grandes centros urbanos y localidades salitreras. De acuerdo con el patrón de vinculaciones se identifican dos comunidades: una centrada en Antofagasta, Calama, Mejillones, Chuquicamata y las oficinas María Elena, Cecilia y Estación Yungay. La segunda centrada en Pampa Unión incluye comunidades secundarias como San Pedro de Atacama, Ollagüe, Pedro de Valdivia, Coya Sur y Bonasort. El pueblo de Pampa Unión, erigido en 1911, fue el gran emporio de las oficinas salitreras no solo del cantón central sino de los cantones del Toco y Aguas Blancas, propicio para el comercio ambulante, un rasgo de los árabes. Los ciclos de bonanza y depresión del salitre afectaron la movilidad mercantil árabe pues hicieron variar las condiciones de vida en el desierto de Atacama. 
Gráfico 5. Localidades de oficinas salitreras y centros urbanos

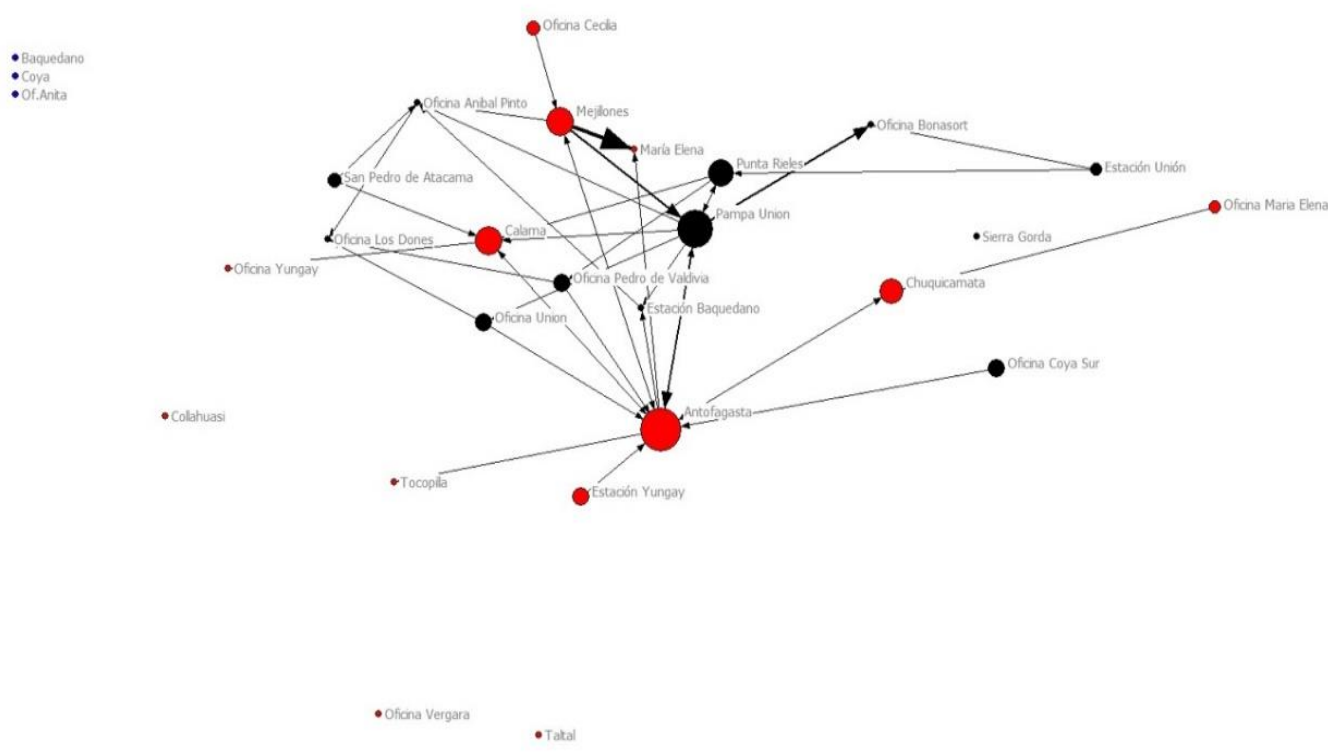

Fuente. Elaboración propia en software Netdraw, a partir de una matriz de localizaciones de los contactos señalados en los prontuarios de los residentes en la Provincia de Antofagasta durante el periodo de estudio.

\section{Los árabes en la región de Antofagasta. Localización en la ciudad de Antofagasta: Análisis de patrones de puntos marcados y redes de vecinos}

En esta sección se concentra la atención solo en los residentes en la ciudad de Antofagasta, por ser ella la que concentra un mayor número de casos y en consecuencia ser el nodo central en el sistema de vinculaciones territoriales derivados del patrón de interacción social declarados por las personas en los prontuarios.

Utilizando la información de la dirección declarada por la persona en el prontuario y realizando una georreferenciación ajustada al sistema de calles y numeración histórica relevante para el periodo de referencia, es posible localizar a las personas en el espacio geográfico urbano utilizando un sistema de coordenadas UTM. En el Mapa 2 se representa la ciudad de Antofagasta contemporánea y en los puntos se sitúan las personas migrantes históricas, él área encerrada en el círculo delimita la ciudad histórica vigente hacia el principio del siglo XX, donde se concentró la población residente bajo análisis. 


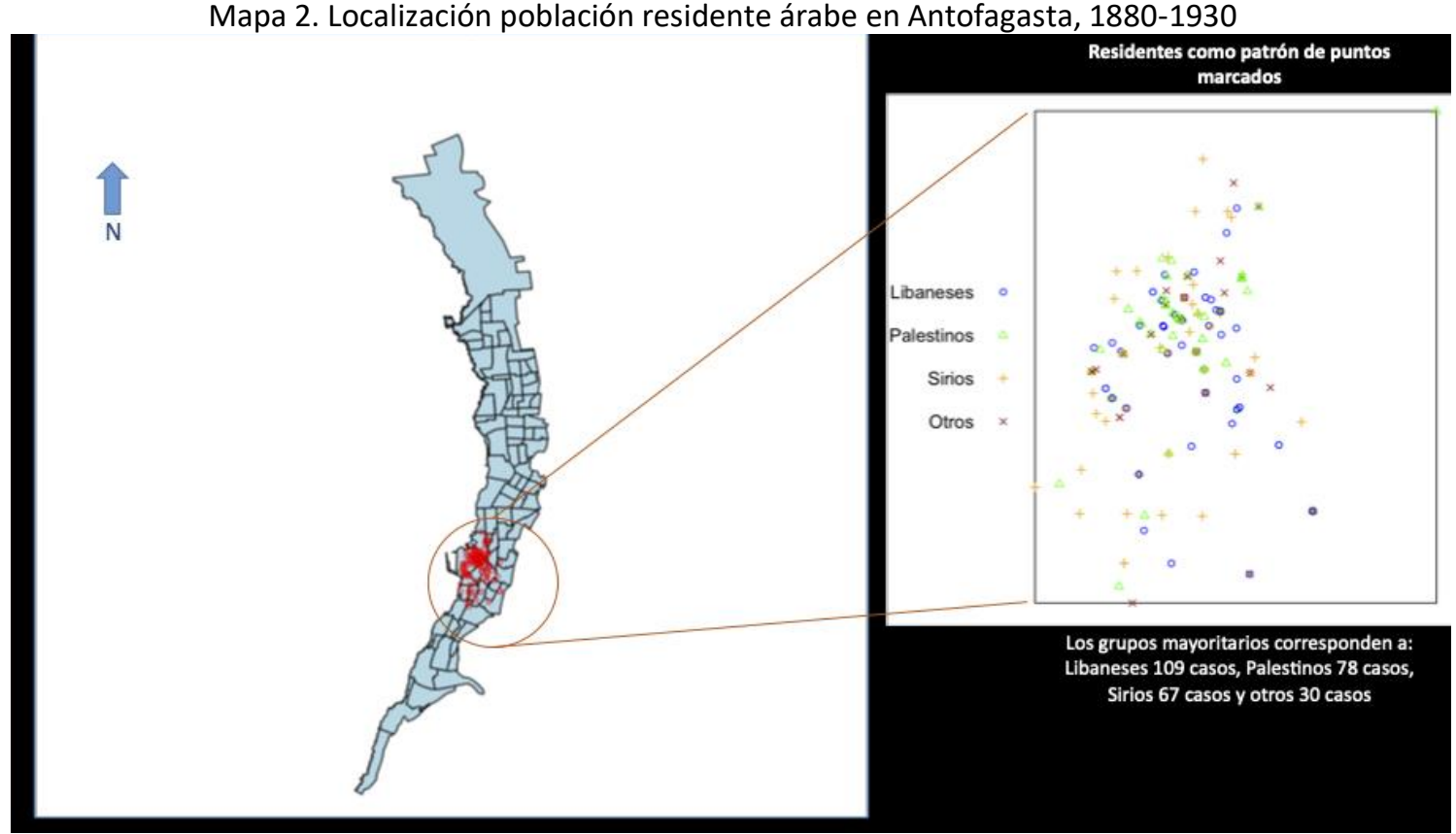

Fuente. Elaboración propia en paquete "MapTools" de R, a partir de una matriz de las direcciones señalados en los prontuarios de los residentes en la Provincia de Antofagasta durante el periodo de estudio (ajustada a los planos históricos relevantes).

Una vez localizadas las personas en el espacio geográfico se procedió a evaluar si el patrón observado correspondía a un tipo de patrón aglomerado, es decir, que las personas tienden a ubicarse más cerca de otras personas, en este caso de personas de su misma "colonia", esta idea es compatible con la configuración de barrios étnicos ${ }^{70}$.

Las pruebas se desarrollaron siguiendo los procedimientos sugeridos por Baddeley \&Turner ${ }^{71}$ y los resultados rechazan la hipótesis de una distribución de puntos que sea completamente aleatoria ${ }^{72}$, luego se realizaron pruebas específicas para probar la hipótesis que el patrón era de tipo aglomerado ${ }^{73}$, separando a los grupos por nacionalidades (libaneses, palestinos, sirios y otros). Los resultados indican que la distribución acumulada de las distancias observadas se concentra en menores distancias que al compararla con el proceso teórico simulado, por lo que las personas de los diferentes grupos nacionales tendieron a colocalizarse

\footnotetext{
70 Logan, John; Alba, Richard y Zhang Wenguan. 2002. "Immigrant Enclaves and Ethnic Enclaves in New York and Los Angeles", en American Sociological Review, № 115, Washington, pp. 299-322.

${ }^{71}$ Baddeley, Adrian y Turner, Rolf. 2005. "Spatstat: An R package for analyzing spatial point patterns", en Journal of Statistical Software, Vol. 12, № 6, Crawley, pp. 1-42.

72 Chi1 $=834.66$, pvalor=2.2e-10, df=24 y también con una prueba basada en MonteCarlo con 999 simulaciones, chi2 $=834.66$, pvalor $=0.002$.

${ }^{73}$ Se utilizó un conjunto de estadísticos tipo K cruzada de Ripley, siguiendo a Baddeley \& Turner. 2005.
} 
(estar cerca) de personas similares. Este rasgo no cambia cuando se comparan los grupos nacionales entre ellos, resultado que sería compatible con la idea de un vecindario árabe no segregado por subgrupos nacionales. Esto también se evalúa dividiendo la muestra por antigüedad de la migración, puesto que la presencia de residentes antiguos podría ser un factor de atracción de nuevos residentes en dichas áreas. Esto ha sido destacado en la literatura sobre migraciones, donde la mayor presencia histórica de inmigrantes actuó como un factor pull ${ }^{74}$, puesto que esta condición afectó la distribución de oportunidades de trabajo y sería determinante del grado de heterogeneidad étnica de las comunidades ${ }^{75}$. El resultado mostró también un patrón aglomerador, es decir los residentes migrantes nuevos tendieron a colocalizarse donde hubo residentes migrantes más antiguos.

Una vez comprobado que el patrón sigue una lógica de vecindario multi-árabe, se pudo explorar la configuración de los vecindarios locales. Para esto se utilizó un criterio de vecindad basado en radios de distancia. De esta forma, para el conjunto de observaciones se definió una red social que representa la relación de "ser vecino de", donde vecino es aquel que reside en un radio de 100 metros. Esto puede ser sostenido en base a la teoría de "Propinquity"76.

La red social de vecinos en el radio espacial puede ser caracterizada en términos de su cohesividad intra e intergrupos nacionales, para esto se utilizó el conjunto de indicadores de segregación propuestos por Reardon \& O' Sullivan ${ }^{77}$.

Los resultados de estos indicadores señalan que los vecindarios tienen una disimilitud muy baja, lo que implica que los vecindarios tienden a ser diversos en términos de su composición por grupos nacionales árabes.

En el gráfico 6 se presenta la red social definida a partir del criterio de vecindad antes explicado, en ella el color del nodo permite identificar la nacionalidad específica del residente.

\footnotetext{
74 Zavodny, Madeline. 1999. "Determinants of recent immigrants locational choices", en International Migration Review, Vol. 33, № 4, Los Angeles (CA), pp. 1014-1030; Belanger, Alain y Rogers Andrei. 1992. "The internal migration and spatial redistribution of the foreign-born population in the United States: 1965-70 and 1975-80", en International Migration Review, $\mathrm{N}^{\circ} 26$, Los Angeles (CA), pp. 1342-1369.

75 O'Loughlin, J. 1995. "The geographic distribution of foreigners in West Germany", en Regional Studies, Vol. 19, № 4, Falmer-Brighton, pp. 365-377; Clark, W. A. 1996. "Scale effects in international migration to the United States", en Regional Studies, Vol. 30, № 6, Falmer-Brighton, pp. 589-600.

76 Blake, Robert; Rhead, Clifton; Wedge, Bryant y Mouton, Jane, 1956. "Housing Architecture and Social Interaction", en Sociometry, Vol. 19, № 2, Washington, pp.133-139.

77 Reardon, Sean; O’Sullivan, David. 2004. “Measures of spatial segregation”, en Sociological Methodology, № 34, Washington, pp. 121-162.
} 
Gráfico 6. Red social y vecindad en la población árabe

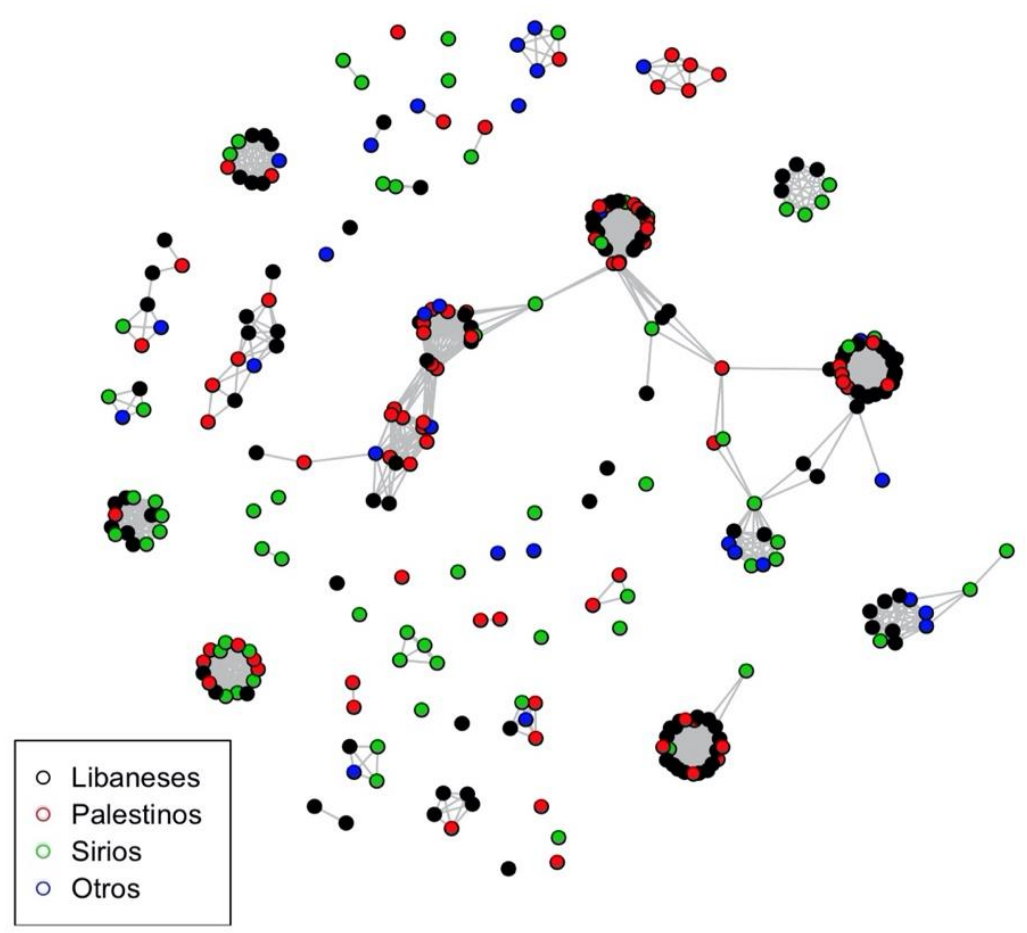

Fuente. Elaboración propia en paquete "sna" de R, a partir de una matriz de vecindarios determinados a partir de las direcciones señaladas en los prontuarios de los residentes en la Provincia de Antofagasta durante el periodo de estudio (ajustada a los planos históricos relevantes).

Estructuralmente esta red tiene un tamaño de 284 localizaciones (personas), con una densidad de 0.039 , lo que implica baja conectividad. Con un degree medio de 11.32 lo que implica que en promedio cada residente árabe encuentra 11 vecinos árabes en un círculo de radio $=100$ metros a su alrededor. También para cada residente en la red (nodo de la red) se calculó una serie de coeficientes de localización de grupos nacionales, además de un indicador de diversidad de la red (como inverso aditivo de un índice de Hirschman-Herfindahl). En el gráfico 7 se muestra los resultados promedios organizados por nacionalidad del nodo. Se identifica que los grupos Sirio y Palestino tienen una colocalización endogrupal (coef.Loc. > 1) más intensa que los libaneses u otros grupos nacionales. 
Gráfico 7. Nodos de red y nacionalidad

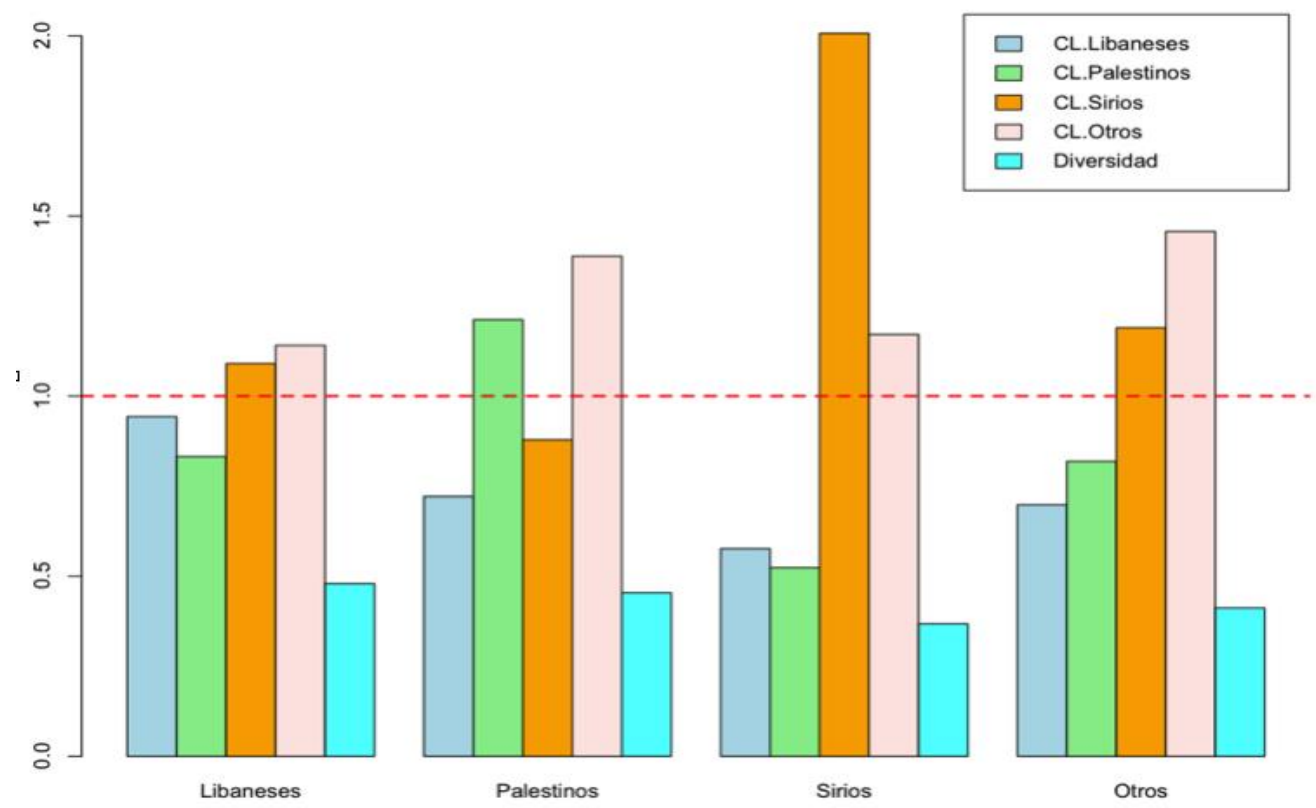

Fuente. Elaboración propia en paquete "sna" de R, a partir de una matriz de vecindarios determinados a partir de las direcciones señaladas en los prontuarios de los residentes en la Provincia de Antofagasta durante el periodo de estudio (ajustada a los planos históricos relevantes).

Para probar que este resultado fuese estadísticamente significativo se realizó una prueba Permanova ${ }^{78}$ siguiendo a Anderson ${ }^{79}$, esta prueba finalmente indica que efectivamente la nacionalidad estableció diferencias en las medias de los coeficientes de localización, lo que implicó que aunque los vecindarios tendieron a ser muy diversos y similares en su composición entre las diferentes grupos, en el caso de los sirios y palestinos, tendió a configurar vecindarios más intensamente compuestos por sujetos de su misma nacionalidad, es decir, existió una suerte de etnificación pero dentro de un marco diverso en general.

\section{Conclusiones preliminares}

La inmigración árabe comenzó a ser visible en la región de Antofagasta, a partir de 1907, coincidiendo con una fuerte bonanza salitrera que, posiblemente, con la ofensiva comercial del

\footnotetext{
78 La prueba PERMANOVA es más adecuada al caso porque no supone una distribución normal, permite la variabilidad grupal, es insensible a la multicolinealidad y al exceso de ceros. La prueba aplicada indica un efecto significativo de la nacionalidad ( $F=4.9454$, pvalor 1e-04), lo que implica que los coeficientes de localización cambian grupo a grupo de nacionalidades.

79 Anderson, Marti. 2001. "A new method for non-parametric multivariate analysis of variance", en Austral Ecology, Vol. 26, № 1, Queensland, pp. 32-46.
} 
producto, posibilitó el conocimiento de la región en áreas del Imperio Turco. Se organizaron los resultados principales de este trabajo en torno a hallazgos que han sido documentados $y$ probados por el conjunto de técnicas estadísticas aplicadas a la información histórica.

En primer lugar ¿por qué vinieron los árabes durante este periodo? La evidencia apuntó a que se trató de una protomigración tipo Sur-Sur, puesto que partieron de una zona de origen ubicada en la periferia de un imperio central decadente hacia una zona "conquistada" en la periferia de una república periférica (la Provincia de Antofagasta, que se incorporó a formalmente a Chile a partir del tratado de Paz y Amistad en 1904). Fue un grupo que sufrió diversas formas de exclusión cultural, económica y religiosa de origen que, en el caso de Antofagasta, por constituir un espacio cosmopolita donde cerca del $20 \%$ de su población en 1907 era extranjera, no fue tan compulsivo como en el centro sur del país. Los análisis empíricos demostraron que, ante la difícil acogida en la sociedad receptora, donde la influencia de las ideas del darwinismo social se hizo sentir, la colonia árabe tendió a reforzar sus patrones históricos de resistencia, cohesión e identidad, configurando vecindarios espacialmente cohesivos y a legitimarse por medio de la actividad comercial. Esto se percibió más nítidamente al poder seguirse la trayectoria personal de cada inmigrante árabe llegado a la provincia de Antofagasta.

En términos de la magnitud de estos flujos migratorios, se trató de una comunidad extranjera poco relevante en su magnitud, pero constante en su crecimiento. En 27 años triplicaron su presencia en Chile (árabes: 1907, 1.177 a 1930, $3.166 \mathrm{v} / \mathrm{s}$ extranjeros: 1907:134.524 a 1930: 105.463), ubicándose en la zona central, la capital y en las ciudades portuarias.

En 1930 la Provincia de Antofagasta aportó el 4\% de la población nacional [4.083.279], pero el $11 \%$ del total de extranjeros residentes en país. Su instalación siguió un proceso que reprodujo la lógica centro-periferia: primero en Santiago y luego en las provincias, así como en la provincia de Antofagasta, es la ciudad de Antofagastav/s cantones salitreros, y en ellos, es Pampa Unión v/s oficinas del cantón central ${ }^{80}$.

En cuanto a su localización en la ciudad, siguió un patrón no aleatorio a nivel general y controlando (marcas) por nacionalidades y por antigüedad de la residencia en el país. El patrón de localización fue "agrupado", configurando un barrio de tipo "multiárabe", no segregado por grupos nacionales de origen, sino que colocalizados y donde los vecinos antiguos atraen vecinos nuevos.

En términos de las redes de vecindarios, construidas en base a vecindarios de 100 metros ("propinquity theory") mostraron baja desigualdad en su composición nacional, pero la

\footnotetext{
80 Hasta 1930 en la Provincia de Antofagasta se registran 1229 casos acumulados, donde la ciudad de Antofagasta concentra el $72 \%$ de ellos.
} 
composición relativa de las redes, usando el Coeficiente de Localización cambió según la nacionalidad del nodo y existió evidencia que para los sirios y palestinos tuvieron una colocalización endo-grupal mayor que para libaneses y otros grupos nacionales.

La inmigración árabe también se vio afectada por la crisis salitrera y mundial de 1929-1930. Los efectos paralizaron el flujo migratorio en el espacio estudiado y dispersaron determinados núcleos establecidos hacia el centro del país.

\section{Referencias citadas}

Archivo Histórico. Ministerio de Relaciones Exteriores de Chile: Vol. 57 (Años 1877-1878), 110 b (Año 1927); 921-A (Año 1922)

Archivo Histórico del Servicio del Registro Civil e Identificaciones. 1880-1930. "Cajas de Prontuarios de Turcos, Palestinos, Libaneses, Sirios", en Archivo Histórico de la Universidad Católica del Norte, Antofagasta, Cajas 154, 164, 165, 178, 179, 183.

Agar, Lorenzo. 1983. “El comportamiento urbano de los migrantes árabes en Chile”, en Eure, Vol. IX, № 27, Santiago, pp. 73-84.

Agar, Lorenzo y Saffie, Nicole. 2005. “Chilenos de origen árabe: la fuerza de las raíces”, en MEAH, Sección Árabe-Islam, № 54, Granada, pp. 3-27.

Akarli, Engin. 1993. The long peace: Ottoman Lebanon, 1861-1920, Berkeley, University of California Press.

Alfaro-Velcamp, Theresa. 2007. So Far from Allah, So Close to Mexico: Middle Eastern Immigrants in Modern Mexico, Austin, University of Texas Press.

Alvarez- Apse, Valentina Mar. 2017. La comunidad libanesa en Chile. Un estudio sobre la migración, cultura e identidad de los descendientes libaneses, Universidad de Bergen Tesis de Maestría, Bergen.

Anderson, Marti J. 2001. "A new method for non-parametric multivariate analysis of variance", en Austral Ecology, Vol. 26, № 1, Queensland, pp. 32-46.

Andrade Chandía, Javiera. 2018. Inmigración árabe en la primera mitad del siglo XX, Universidad Católica de la Santísima Concepción, Tesis Licenciatura en Historia.

Ministerio del Interior. 1909. Anuario correspondiente al año 1906, Santiago de Chile, Imprenta Nacional.

Araneda Tapia, Jorge. 2015. "Las ilusiones y los padecimientos de la emigración, el caso de los inmigrantes árabes musulmanes levantinos a Chile 1930-1950", en Tabula Rasa, № 22, Bogotá, pp. 125-146.

Baddeley, Adrian y Turner, Rolf. 2005. "Spatstat: An R package for analyzing spatial point patterns", en Journal of Statistical Software, Vol. 12, № 6, Crawley, pp. 1-42.

Belanger, Alain y Rogers, Andrei. 1992. "The internal migration and spatial redistribution of the foreignborn population in the United States: $1965-70$ and 1975-80", en International Migration Review, $\mathrm{N}^{\circ}$ 26, Los Angeles (CA), pp. 1342-1369. 
Blake, Robert, Rhead, Clifton, Wedge, Bryant y Mouton, Jane Srygley. 1956. "Housing Architecture and Social Interaction", en Sociometry, Vol. 19, № 2, Washington, pp.133-139.

Blakemore, Harold. 1996. Historia del Ferrocarril de Antofagasta a Bolivia. 1888-1988, Santiago, Impresos Universitarios S.A.

Bonacich, Phillip. 2007. "Some unique properties of eigenvector centrality", en Social Networks, Vol. 29, № 4, Laussane, pp. 555-564.

Borgatti, Sthephen y Everett, Martin. 1997. "Network analysis of 2-mode data”, en Social Networks, Vol. 19, № 3, Laussane, pp. 243-269.

Breiger, Ronald. 1974. "The Duality of Persons and Groups", en Social Forces, Vol. 53, № 2, Chapel Hill, pp. 181-190.

Campbell, Karen y Lee, Barrett. 1991. "Name generators in surveys of personal networks", en Social Networks, № 13, Laussane, pp. 203-221.

Cano, Verónica y Soffia, Magdalena. 2009. "Los estudios sobre migración internacional en Chile: apuntes y comentarios para una agenda de investigación actualizada", en Papeles de Migración, № 61, Ciudad de México, pp. 129-167.

Cánovas, Rodrigo. 2011. "Letras judías y árabes en Chile: Otros cobijos", en América sin nombre, no 16, Alicante, pp. 131-140.

Oficina Central de Estadística. Censos Nacionales de Población de Chile de 1885, 1895, 1907, 1920 y 1930. Disponible en Memoria Chilena: http://www.memoriachilena.gob.cl/602/w3-article31530.html\#documentos (Consultado el 26 de julio de 2020).

Clark, Williams. 1996. "Scale effects in international migration to the United States", en Regional Studies, Vol. 30, № 6, Falmer-Brighton, pp. 589-600.

Crisóstomo Ayres, Stefano. 2018. Espacios, dinámica e integración: el caso de la comunidad musulmana en Iquique (1999-2018), Universidad de Chile, Santiago, Informe de Seminario para optar al grado de Licenciado en Historia.

Chahuán, Eugenio. 1983. "Presencia árabe en Chile", en Revista Chilena de Humanidades, № 4, Santiago, pp. 33-45.

Chase-Dunn, Christopher y Hall, Thomas. 1994. "The historical evolution of world-systems", en Sociological Inquiry, Vol. 64, № 3, Los Angeles (CA), pp. 257-280.

Daraganova, Galina, Pattison, Pip, Koskinen, Johan, Mitchell, Bill, Bill, Anthea, Watts, Martin y Baum, Scott. 2012. "Networks and geography: modelling community network structures as the outcome of both spatial and network processes", en Social Networks, Vol. 34, № 1, Laussane, pp. 6-17.

Díaz Aguad, Alfonso. 2017. "Inmigración árabe en el área Centro Sur Andina” en Díaz, Alfonso. Ser Inmigrante entre el Sama y El Loa. Integración económica y social en un espacio de frontera, Arica, Ediciones de la Universidad de Tarapacá, pp.15-26.

Estrada, Baldomero. 2014. "Desarrollo empresarial inmigrante. La colectividad árabe en Valparaíso, Chile (1900-1940)", en Interciencia, Vol. 39, № 12, Caracas, pp. 850-856. 
Estrada, Baldomero. 2017. "Integración laboral y social de las colectividades árabes en las ciudades medianas de chile durante el siglo xx: el caso de Quillota", en Historia 396, № 1, Viña del Mar, pp. 5987.

Fahrenthold, Stacy. 2019. "Arab Labor Migration in the Americas, 1880-1930", en American History, in Oxford Research Encyclopedias. Online Publication. Date: May 2019. Disponible en: https://oxfordre.com/americanhistory/view/10.1093/acrefore/9780199329175.001.0001/acrefore9780199329175-e-598 (Consultado el 15 de mayo de 2020).

Fassmann, Heinz y Münz, Rainer. 1992. "Patterns and trends of international migration in Western Europe", en Population and Development Review, Vol. 18, № 3, Nueva York, pp. 457-480.

Fawcett, James y Arnold, Fred. 1987. "19: Explaining Diversity: Asian and Pacific Immigration Systems", en Center for Migration Studies, Vol 5, №3, New York, pp. 453-473.

Fersan, Eliane. 2010. "Syro-Lebanese migration (1880-present): 'push' and "pull' factors", en Working Paper Middle East Institute, Washington DC., USA. Disponible en https://www.mei.edu/publications/syro-lebanese-migration-1880-present-push-and-pull-factors. (Consultado el 24 de Julio de 2020).

Fuenzalida, Humberto. 1950. “Clima”, en Geografía Económica de Chile, Santiago, Imprenta Universitaria, Tomo I, pp. 188-257.

Ghosh, Bimal. 1992. "Migration, trade and international economic cooperation. Do the interlinkages work?", en International Migration, Vol. 30, №s 3-4, Geneve, pp. 377-398.

Girvan, Michelle y Newman, Mark. 2002. "Community structure in social and biological networks", en Proceedings of the National Academy of Sciences, Vol. 99, № 12, Washington, pp. 7821-7826.

González Pizarro, José Antonio. 1989. “España y la guerra con Marruecos, 1859-1860. Política interna, proyección exterior y repercusiones en Iberoamérica”, en Estudos Iberoamericanos, Vol. XV, № 2, Porto Alegre, pp. 369-381.

González Pizarro, José Antonio. 2008. "La conquista de una frontera. Mentalidades y tecnologías en las vías de comunicación en el desierto de Atacama", en Revista de Geografía. Norte Grande, N 40, Santiago, pp. 23-46.

González Pizarro, José Antonio. 2016. La épica del salitre en el desierto de Atacama: 1880-1967. Trabajo, tecnologías, vida cotidiana, conflicto y cultura, Saarbrücken, Editorial Académica Española.

González Pizarro, José Antonio. 2020 "Desde la influencia del darwinismo social hasta el imperio de los derechos humanos. Inmigración en Chile entre 1907 y 2018", en Estudios de Derecho, № 169, Antioquía, pp. 325-348.

González Pizarro, José Antonio, Lufin Varas, Marcelo y Galeno Ibaceta, Claudio. 2018. "La migración de las colonias minoritarias europeas y latinoamericanas en el desierto de Atacama. Españoles, italianos, colombianos y ecuatorianos en Antofagasta, 1880-1930", en Tapia Ladino, Marcela y Liberona Concha, Nanette (eds.), El afán de cruzar las fronteras. Enfoques transdisciplinarios sobre migraciones y movilidad en Sudamérica y Chile, Santiago, RIL Editores-Universidad Arturo Prat, pp. 223-257.

Goodwin, Jason. 2016. Los señores del Horizonte. Una historia del Imperio otomano, Madrid, Alianza Editorial.

Hourani, Albert. 2008. La historia de los árabes, Editorial Zeta, Barcelona. 
Houari, Saim. 2016-2017. Lo árabe en la literatura hispanoamericana: Sergio Macías, Benedicto Chuaqui y Jorge García Usta, Université d’Oran 2, Orán, Tesis para optar al grado de Doctor en Ciencias de la Lengua Española.

Hyland Jr, Steven. 2011. "'Arisen from deep slumber': transnational politics and competing nationalisms among syrian immigrants in Argentina, 1900-1922", en Journal of Latin American Studies, N 43, Cambridge, pp. 547-574.

Hyland Jr, Steven L. 2011a. Margins of the Mahjar: arabic-speaking immigrants in Argentina, 1880-1946, Ohio, The Ohio State University. Disponible en https://etd.ohiolink.edu/apexprod/rws_olink/r/1501/10?clear=10\&p10_accession_num=osu13065 10917 (Consultado el 18 de julio de 2020).

Hyland, Steven. 2016. "Arabic-speaking Immigrants before the Courts in Tucumán, Argentina, 19101940", en Journal of Women's History, Vol. 28, № 4, Baltimore, pp.41-64.

Hyland Jr, Steven. 2018. "The Syrian-Ottoman home front in Buenos Aires and Rosario during the First World War", en Journal of Migration History, Vol. 4, № 1, Leiden, pp. 211-235.

Ilyas, Muhammad y Radha, Hayder. 2011. "Identifying influential nodes in online social networks using principal component centrality", en 2011 IEEE International Conference on Communications, New York, pp. 1-5.

Karpat, Kemal. 1985. Ottoman population, 1830-1914: demographic and social characteristics, Wisconsin, The University of Wisconsin Press.

Kévorkian, Raymond. 2011. The Armenian genocide. A complete history, London, I. B. Tauris

Khater, Akram Fouad. 2001. Inventing home: emigration, gender, and the middle class in Lebanon, $1870-$ 1920. Oakland, University of California Press. Disponible en http://ark.cdlib.org/ark:/13030/ft9d5nb66k/ (Consultado el 14 de junio de 2020).

Klich, Ignacio. 1993. "Argentina-Ottoman. relations and their Impact in Immigrants from the Middle East: a history unfulfilled expectations, 1910-1915", en The Americas, Vol. 50, № 2, Cambridge, pp. 177205.

Klich, Ignacio y Lesser, Jeffrey. 1996. "Introduction 'Turco' immigrants in Latin America”, en The Americas, Vol.53, N 1, jul, Cambridge, pp. 1-14.

Krämer, Gudrun. 2006. Historia de Palestina. Desde la conquista otomana hasta la fundación del estado de Israel, Madrid, Siglo XXI.

Kukovecz, Gyorgy "The "Turks" of Latin America: remarks of the problems of Syrian- Lebanese emigration", Acta. bibli. u-szeged. hum. Disponible en https://www.semanticscholar.org/paper/The\%22Turks\%22-of-Latin-America-\%3A-remarks-on-the-ofKukovecz/de4d898e611a4f2b3d33fbd8801fa0d2d87d674c (Consultado el 17 de julio de 2020).

Logan, John, Alba, Richard y Wenquan, Zhang. 2002. "Immigrant Enclaves and Ethnic Enclaves in New York and Los Angeles", en American Sociological Review, № 115, Washington, pp. 299-322.

Marin, Alexandra y Hampton, Keith. 2007. "Simplifying the personal network name generator", en Field Methods, № 19, Los Angeles (CA), pp. 163-193. 
Mattar, Ahmad Hassan. 1941. Guía Social de la Colonia Árabe en Chile (Siria-Palestina-Libanesa), Santiago, Imprenta Ahues Hnos.

Ministerio de Relaciones Exteriores de Chile. Relaciones Bilaterales, Embajada de Chile en Turquía. Disponible en https://chile.gob.cl/turquia/relacion-bilateral/relaciones-bilaterales (Consultado el 23 de junio de 2020).

Nasser, Ghinwa. 2015. Inmigración, identidad y estrategias de adaptación a la sociedad receptora. El caso de las mujeres sirias y libanesas en Argentina (primera mitad del siglo XX), Departamento de Historia de América. Programa de Doctorado en Historia, Literatura y poder. Procesos interétnicos y culturales en América, Universidad de Sevilla, Tesis para optar al grado de Doctor.

O'Loughlin, John. 1995. "The geographic distribution of foreigners in West Germany", en Regional Studies, Vol. 19, № 4, Falmer-Brighton, pp. 365-377.

Olguín Tenorio, Myriam y Peña González, Patricia. 1990. La inmigración árabe en Chile, Santiago, Ediciones Instituto Chileno Árabe de Cultura, pp. 104-108.

Pacheco, Juan Antonio. 2006. "La prensa árabe en Chile: sueños y realidades árabes en un mundo nuevo", en MEAH, Sección Árabe-Islam, № 55, Granada, pp. 277-322.

Portes, Alejandro y Böröcz, Jözsef. 1989. "Contemporary immigration: theoretical perspectives on its determinants and modes of incorporation", en International Migration Review, Vol. 23, № 3, Los Angeles (CA), pp. 606-630.

Pustejovsky, James y Spillane, James. 2009. "Question-order effects in social network name generators", en Social Networks, № 31, Laussane, pp. 221-229.

Qiao, Shaojie, Li, Tianrui, Li, Hong, Peng, Jing y Chen, Hongmei. 2012. "A new blockmodeling based hierarchical clustering algorithm for web social networks", en Engineering Applications of Artificial Intelligence, Vol. 25, № 3, Amsterdam, pp. 640-647.

Rafide, Matías. 1989. Escritores chilenos de origen árabe, Santiago Instituto Chileno-Árabe de Cultura, pp. 87-110.

Reardon, Sean y O'Sullivan, David. 2004. "Measures of spatial segregation”, en Sociological Methodology, № 34, Washington, pp. 121-162.

Rebolledo Hernández, Antonia. 1994. "La "turcofobia”. Discriminación antiárabe en Chile. 1900-1950", en Historia, № 28, Santiago, pp. 249-272.

Rogan, Eugene. 2018. Los árabes. Del Imperio otomano a la actualidad, Barcelona, Editorial CríticaPlaneta.

Said, Bahajin. 2008. "El modelo latinoamericano en la integración de los inmigrantes árabes", en $R a$ Ximhai, Vol. 4, № 3, Ciudad de México, pp. 737-773.

Samamé, María Olga. 2003. "Transculturación, identidad y alteridad en novelas de la inmigración árabe hacia Chile", en Signos, Vol. 36, № 53, Valparaíso, pp. 51-73.

Sanfuentes, Andrés. 1964. La influencia de los árabes en el desarrollo económico de Chile, Memoria de Título, Santiago, Universidad de Chile. 
Solórzano Morán, Claudia. 2015. La migración palestina hacia el Ecuador y su influencia en la sociedad y política exterior ecuatoriana, Universidad Internacional del Ecuador, Quito, Trabajo de Investigación para la obtención del título de Internacionalista.

Tapia, Marcela y Liberona, Nanette (Editoras). 2018. El afán de cruzar las fronteras. Enfoques transdisciplinarios sobre migraciones y movilidad en Sudamérica y Chile. RIL Editores-Universidad Arturo Prat,

Tarazi-Fawaz, Leila. 1983. "Merchants and Migrants in Nineteenth-Century Beirut", en Harvard Middle Eastern Studies, № 18, Cambridge, pp. 2-3.

Thomson, Ian. 2003. La Red Norte: la historia de los ferrocarriles del norte chileno, Santiago, Imprenta Silva.

Viera Núñez, Fabiola. 2014. Relaciones diplomáticas de Chile hacia países árabes: casos de Siria y Egipto (2005-2011), Universidad de Chile, Santiago, Tesis para optar al Grado de Magister en Estudios Internacionales.

Zavodny, M. 1999. "Determinants of recent immigrants locational choices", en International Migration Review, Vol. 33, № 4, Los Angeles (CA), pp. 1014-1030. 